\title{
Time-Resolved Infrared (TRIR) Studies of Oxycarbonylnitrenes
}

\section{Supporting Information}

Tyler A. Chavez, Yonglin Liu, and John P. Toscano*

Department of Chemistry, Johns Hopkins University, Baltimore, Maryland 21218, United States

jtoscano@jhu.edu

\section{Contents:}

Tables S1-S52: Optimized geometries and energies 2

Figure S1: Calculated rotation barrier for 2s 41

Figure S2: Calculated rotation barrier for $\mathbf{2 t} \quad 42$

Figure S3: Calculated rotation barrier for $4 s \quad 43$

Figure S4: Calculated rotation barrier for 4t 44

Figure S5: Observed kinetics at $1640 \mathrm{~cm}^{-1}$ in the presence of TES 44

Compound characterization: ${ }^{1} \mathrm{H}$ and ${ }^{13} \mathrm{C}$ NMR spectra of final compounds $\quad 45$

Photolysis product studies: ${ }^{1} \mathrm{H}$ NMR spectra of $\mathbf{6}$ and $\mathbf{7}$ after photolysis $\quad 47$ 
Table S1 B3LYP/6-31G* optimized geometries, energies, and thermal corrections for the syn-singlet Ethoxycarbonylnitrene $\mathbf{2} \mathbf{s}_{\text {syn }}$

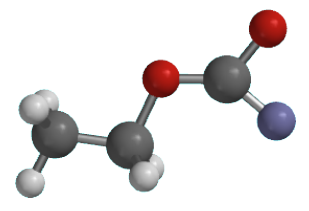

Dipole Moment B3LYP/6-31G* energy Zero-point correction Thermal correction to energy

\author{
4.55 Debye \\ -322.402226 Hartrees \\ 0.082710276 Hartrees \\ 0.089593919 Hartrees
}

\begin{tabular}{ccrrr}
\hline Atom & $\begin{array}{c}\text { Atomic } \\
\text { Number }\end{array}$ & \multicolumn{2}{c}{ Cartesian Coordinates } \\
$\mathbf{X}$ & $\mathbf{Y}$ & \multicolumn{1}{c}{$\mathbf{Z}$} \\
\hline $\mathrm{O}$ & 8 & 0.1849282 & -0.5839627 & 0.0000000 \\
$\mathrm{O}$ & 8 & -1.5846722 & 0.9877074 & 0.0000000 \\
$\mathrm{~N}$ & 7 & -2.2155665 & -0.7373761 & 0.0000000 \\
$\mathrm{C}$ & 6 & 2.5444169 & -0.1759165 & 0.0000000 \\
$\mathrm{C}$ & 6 & 1.1834965 & 0.4882771 & 0.0000000 \\
$\mathrm{C}$ & 6 & -1.0634316 & -0.1905919 & 0.0000000 \\
$\mathrm{H}$ & 1 & 1.0243431 & 1.1051511 & -0.8891371 \\
$\mathrm{H}$ & 1 & 1.0243431 & 1.1051511 & 0.8891371 \\
$\mathrm{H}$ & 1 & 3.3244967 & 0.5926194 & 0.0000000 \\
$\mathrm{H}$ & 1 & 2.6734220 & -0.8009292 & -0.8887391 \\
$\mathrm{H}$ & 1 & 2.6734220 & -0.8009292 & 0.8887391 \\
\hline
\end{tabular}

Table S2. B3LYP/6-31 $\mathrm{G}^{*}$ calculated IR frequencies $\left(\mathrm{cm}^{-1}\right.$, unscaled) and intensities for syn-singlet Ethoxycarbonylnitrene $\mathbf{2} \mathbf{s}_{\text {synn }}$.

\begin{tabular}{lrlr}
\hline Frequency & Intensity & Frequency & Intensity \\
\hline 34 & 1.63 & 1311 & 0.53 \\
118 & 0.21 & 1411 & 77.13 \\
181 & 2.35 & 1413 & 25.43 \\
250 & 0.23 & 1452 & 34.43 \\
353 & 15.40 & 1514 & 6.70 \\
448 & 10.67 & 1528 & 3.45 \\
581 & 0.24 & 1546 & 8.25 \\
627 & 29.38 & 1826 & 369.21 \\
825 & 0.76 & 3069 & 9.78 \\
854 & 7.11 & 3086 & 11.13 \\
1020 & 5.08 & 3131 & 1.83 \\
1100 & 200.99 & 3142 & 16.78 \\
1141 & 3.83 & 3155 & 30.15 \\
1188 & 4.55 & & \\
\hline
\end{tabular}


Table S3 B3LYP/6-31G* optimized geometries, energies, and thermal corrections for syn-triplet Ethoxycarbonylnitrene $\mathbf{2} \mathbf{t}_{\text {syn }}$

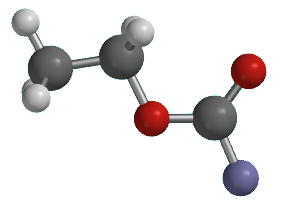

Dipole Moment UB3LYP/6-31G* energy Zero-point correction Thermal correction to energy
3.26 Debye -322.4225538 Hartrees 0.082232539 Hartrees 0.089074246 Hartrees

\begin{tabular}{ccrrr}
\hline Atom & $\begin{array}{c}\text { Atomic } \\
\text { Number }\end{array}$ & \multicolumn{3}{c}{ Cartesian Coordinates (Angstroms) } \\
& $\mathbf{X}$ & \multicolumn{1}{c}{$\mathbf{Y}$} & \multicolumn{1}{c}{$\mathbf{Z}$} \\
\hline $\mathrm{O}$ & 8 & 0.1059323 & -0.5089712 & -0.0081129 \\
$\mathrm{O}$ & 8 & -1.4622653 & 1.1535638 & 0.0069326 \\
$\mathrm{~N}$ & 7 & -2.1171029 & -1.0235922 & 0.0016209 \\
$\mathrm{C}$ & 6 & 2.4870834 & -0.2676857 & 0.0098588 \\
$\mathrm{C}$ & 6 & 1.1702556 & 0.4820998 & -0.0115201 \\
$\mathrm{C}$ & 6 & -1.1410471 & -0.0259254 & 0.0008665 \\
$\mathrm{H}$ & 1 & 2.5818281 & -0.9174423 & -0.8655912 \\
$\mathrm{H}$ & 1 & 2.5717111 & -0.8839294 & 0.9102334 \\
$\mathrm{H}$ & 1 & 3.3163549 & 0.4477312 & 0.0013852 \\
$\mathrm{H}$ & 1 & 1.0590366 & 1.1031920 & -0.9062782 \\
$\mathrm{H}$ & 1 & 1.0437024 & 1.1279209 & 0.8631153 \\
\hline
\end{tabular}

Table S4. B3LYP/6-31 $\mathrm{G}^{*}$ calculated IR frequencies $\left(\mathrm{cm}^{-1}\right.$, unscaled) and intensities for syn-triplet Ethoxycarbonylnitrene $\mathbf{2 t}_{\text {syn. }}$.

\begin{tabular}{lrlr}
\hline Frequency & Intensity & Frequency & Intensity \\
\hline 58 & 0.88 & 1280 & 326.72 \\
150 & 0.35 & 1305 & 0.80 \\
193 & 0.96 & 1413 & 4.18 \\
267 & 0.18 & 1453 & 14.03 \\
361 & 16.10 & 1517 & 6.21 \\
430 & 4.28 & 1525 & 1.82 \\
630 & 5.79 & 1541 & 7.48 \\
682 & 36.00 & 1672 & 201.41 \\
820 & 0.42 & 3066 & 12.37 \\
870 & 7.15 & 3076 & 11.14 \\
989 & 6.95 & 3118 & 5.55 \\
1062 & 86.94 & 3138 & 19.06 \\
1146 & 5.22 & 3148 & 30.89 \\
1188 & 4.46 & & \\
\hline
\end{tabular}


Table S5 B3LYP/6-31G* optimized geometries, energies, and thermal corrections for the anti-singlet Ethoxycarbonylnitrene $\mathbf{2} \mathbf{s}_{\text {anti }}$

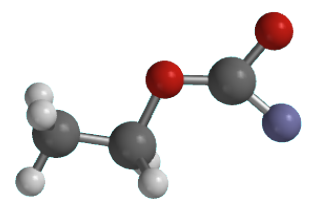

Dipole Moment UB3LYP/6-31G* energy Zero-point correction Thermal correction to energy
4.75 Debye
-322.402280 Hartrees
0.082765276 Hartrees
0.089640424 Hartrees

\begin{tabular}{ccrrr}
\hline Atom & $\begin{array}{c}\text { Atomic } \\
\text { Number }\end{array}$ & \multicolumn{3}{c}{ Cartesian Coordinates } \\
& $\mathbf{X}$ & \multicolumn{1}{c}{$\mathbf{Y}$} & \multicolumn{1}{c}{$\mathbf{Z}$} \\
\hline $\mathrm{O}$ & 8 & 0.1845572 & -0.5205227 & 0.0055243 \\
$\mathrm{O}$ & 8 & -2.1431315 & -0.7176314 & -0.0023593 \\
$\mathrm{~N}$ & 7 & -1.6730702 & 1.0481120 & -0.0027249 \\
$\mathrm{C}$ & 6 & 2.5622626 & -0.2057985 & -0.0045926 \\
$\mathrm{C}$ & 6 & 1.2265364 & 0.5065796 & 0.0037296 \\
$\mathrm{C}$ & 6 & -1.0399068 & -0.0594773 & 0.0011224 \\
$\mathrm{H}$ & 1 & 1.0868542 & 1.1315926 & -0.8844323 \\
$\mathrm{H}$ & 1 & 1.0948411 & 1.1262961 & 0.8968483 \\
$\mathrm{H}$ & 1 & 3.3705360 & 0.5328990 & -0.0038490 \\
$\mathrm{H}$ & 1 & 2.6640873 & -0.8305381 & -0.8969632 \\
$\mathrm{H}$ & 1 & 2.6704131 & -0.8396231 & 0.8805927 \\
\hline
\end{tabular}

Table S6. B3LYP/6-31G* calculated IR frequencies $\left(\mathrm{cm}^{-1}\right.$, unscaled) and intensities for anti-singlet Ethoxycarbonylnitrene $2 \mathbf{s}_{\text {anti }}$.

\begin{tabular}{lrlr}
\hline Frequency & Intensity & Frequency & Intensity \\
\hline 41 & 1.63 & 1312 & 0.95 \\
129 & 0.04 & 1403 & 83.40 \\
169 & 2.75 & 1434 & 22.57 \\
257 & 0.13 & 1458 & 23.55 \\
348 & 11.64 & 1515 & 6.90 \\
472 & 7.52 & 1528 & 3.60 \\
562 & 0.84 & 1546 & 9.41 \\
628 & 28.75 & 1817 & 394.75 \\
828 & 0.97 & 3070 & 8.61 \\
865 & 13.79 & 3073 & 12.59 \\
1022 & 12.70 & 3118 & 8.25 \\
1101 & 156.69 & 3143 & 15.43 \\
1149 & 13.03 & 3152 & 25.03 \\
1190 & 4.64 & & \\
\hline
\end{tabular}


Table S7. B3LYP/6-31G* optimized geometries, energies, and thermal corrections for anti-triplet Ethoxycarbonylnitrene $\mathbf{2} \mathbf{t}_{\text {anti }}$

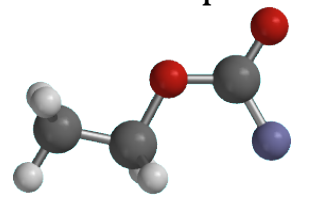

Dipole Moment UB3LYP/6-31G* energy Zero-point correction Thermal correction to energy
3.77 Debye

-322.4162641 Hartrees 0.081918123 Hartrees 0.088766571 Hartrees

\begin{tabular}{ccrrr}
\hline Atom & $\begin{array}{c}\text { Atomic } \\
\text { Number }\end{array}$ & \multicolumn{3}{c}{ Cartesian Coordinates } \\
& $\mathbf{X}$ & \multicolumn{1}{c}{$\mathbf{Y}$} & \multicolumn{1}{c}{$\mathbf{Z}$} \\
\hline $\mathrm{O}$ & 8 & 0.1178635 & -0.5002564 & -0.0053311 \\
$\mathrm{O}$ & 8 & -2.0998488 & -0.8458674 & 0.0017670 \\
$\mathrm{~N}$ & 7 & -1.4251950 & 1.2888565 & 0.0033517 \\
$\mathrm{C}$ & 6 & 2.4956771 & -0.2715079 & 0.0050174 \\
$\mathrm{C}$ & 6 & 1.1843073 & 0.4891813 & -0.0050299 \\
$\mathrm{C}$ & 6 & -1.1504205 & -0.0711407 & 0.0000248 \\
$\mathrm{H}$ & 1 & 2.5786505 & -0.9124753 & -0.8779128 \\
$\mathrm{H}$ & 1 & 2.5731221 & -0.8990422 & 0.8980353 \\
$\mathrm{H}$ & 1 & 3.3325569 & 0.4348759 & 0.0023927 \\
$\mathrm{H}$ & 1 & 1.0891711 & 1.1175667 & -0.8972161 \\
$\mathrm{H}$ & 1 & 1.0813633 & 1.1268736 & 0.8796785 \\
\hline
\end{tabular}

Table S8. B3LYP/6-31G* calculated IR frequencies $\left(\mathrm{cm}^{-1}\right.$, unscaled) and intensities for anti-triplet Ethoxycarbonylnitrene $\mathbf{2} \mathbf{t}_{\text {anti }}$.

\begin{tabular}{lrlr}
\hline Frequency & Intensity & Frequency & Intensity \\
\hline 51 & 0.39 & 1279 & 345.68 \\
129 & 0.45 & 1314 & 0.61 \\
197 & 0.24 & 1412 & 22.05 \\
268 & 0.42 & 1450 & 29.49 \\
353 & 8.63 & 1516 & 6.31 \\
508 & 10.92 & 1526 & 4.70 \\
551 & 11.17 & 1542 & 9.09 \\
656 & 39.12 & 1634 & 216.30 \\
826 & 0.77 & 3063 & 18.54 \\
842 & 14.32 & 3067 & 10.82 \\
984 & 2.82 & 3107 & 12.98 \\
1069 & 56.67 & 3140 & 19.04 \\
1139 & 41.41 & 3149 & 26.61 \\
1186 & 4.55 & & \\
\hline
\end{tabular}


Table S9. B3LYP/6-31G* optimized geometries, energies, and thermal corrections for syn-singlet t-butoxycarbonylnitrene $\mathbf{4} \mathbf{s}_{\text {syn }}$

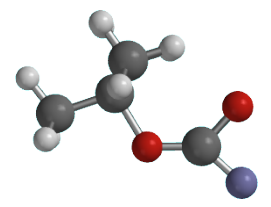

Dipole Moment UB3LYP/6-31G* energy Zero-point correction Thermal correction to energy
4.80 Debye -401.037929 Hartrees 0.138609656 Hartrees 0.147923845 Hartrees

\begin{tabular}{ccrrr}
\hline Atom & $\begin{array}{c}\text { Atomic } \\
\text { Number }\end{array}$ & \multicolumn{3}{c}{ Cartesian Coordinates } \\
& $\mathbf{X}$ & \multicolumn{1}{c}{$\mathbf{Y}$} & \multicolumn{1}{c}{$\mathbf{Z}$} \\
\hline $\mathrm{O}$ & 8 & -0.2371798 & -0.8614080 & 0.0000000 \\
$\mathrm{O}$ & 8 & -1.8493200 & 0.9000120 & 0.0000000 \\
$\mathrm{~N}$ & 7 & -2.6312507 & -0.7387603 & 0.0000000 \\
$\mathrm{C}$ & 6 & 0.9832474 & 0.0100739 & 0.0000000 \\
$\mathrm{C}$ & 6 & 0.9882699 & 0.8533212 & 1.2755995 \\
$\mathrm{C}$ & 6 & 0.9882699 & 0.8533212 & -1.2755995 \\
$\mathrm{C}$ & 6 & 2.1150022 & -1.0142107 & 0.0000000 \\
$\mathrm{C}$ & 6 & -1.4245733 & -0.3229676 & 0.0000000 \\
$\mathrm{H}$ & 1 & 0.9284562 & 0.2104678 & 2.1599027 \\
$\mathrm{H}$ & 1 & 0.1527961 & 1.5573965 & 1.2967731 \\
$\mathrm{H}$ & 1 & 1.9220404 & 1.4233166 & 1.3307840 \\
$\mathrm{H}$ & 1 & 0.1527961 & 1.5573965 & -1.2967731 \\
$\mathrm{H}$ & 1 & 0.9284562 & 0.2104678 & -2.1599027 \\
$\mathrm{H}$ & 1 & 1.9220404 & 1.4233166 & -1.3307840 \\
$\mathrm{H}$ & 1 & 2.0616005 & -1.6502424 & 0.8886284 \\
$\mathrm{H}$ & 1 & 3.0796701 & -0.4966156 & 0.0000000 \\
$\mathrm{H}$ & 1 & 2.0616005 & -1.6502424 & -0.8886284 \\
\hline
\end{tabular}


Table S10. B3LYP/6-31G* calculated IR frequencies $\left(\mathrm{cm}^{-1}\right.$, unscaled) and intensities for syn-singlet $t$-butoxycarbonylnitrene $\mathbf{4} \mathbf{s}_{\text {synn }}$.

\begin{tabular}{lrlr}
\hline Frequency & Intensity & Frequency & Intensity \\
\hline 73 & 0.05 & 1287 & 17.48 \\
104 & 0.44 & 1297 & 12.34 \\
171 & 3.03 & 1393 & 78.13 \\
212 & 0.06 & 1428 & 14.66 \\
262 & 0.00 & 1432 & 16.61 \\
275 & 0.13 & 1458 & 12.39 \\
308 & 4.07 & 1501 & 0.36 \\
343 & 0.45 & 1517 & 0.27 \\
361 & 6.62 & 1521 & 0.02 \\
418 & 3.65 & 1526 & 2.58 \\
451 & 1.27 & 1530 & 4.90 \\
459 & 15.63 & 1553 & 5.62 \\
582 & 1.36 & 1822 & 397.13 \\
630 & 30.38 & 3062 & 6.46 \\
745 & 7.84 & 3062 & 13.57 \\
833 & 18.99 & 3070 & 8.99 \\
937 & 0.07 & 3131 & 5.02 \\
941 & 0.21 & 3132 & 7.39 \\
986 & 0.07 & 3142 & 32.89 \\
1061 & 29.32 & 3144 & 4.63 \\
1067 & 0.60 & 3152 & 16.50 \\
1094 & 133.36 & 3155 & \\
1211 & 92.92 & & \\
\hline
\end{tabular}


Table S11. B3LYP/6-31G* optimized geometries, energies, and thermal corrections for syn-triplet t-butoxycarbonylnitrene $\mathbf{4 t}_{\text {syn }}$

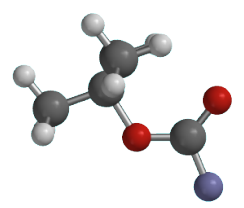

Dipole Moment UB3LYP/6-31G* energy Zero-point correction -401.055453 Hartrees Thermal correction to energy 0.138014188 Hartrees 0.147310667 Hartrees

\begin{tabular}{ccrrr}
\hline Atom & $\begin{array}{c}\text { Atomic } \\
\text { Number }\end{array}$ & \multicolumn{3}{c}{ Cartesian Coordinates } \\
& $\mathbf{X}$ & $\mathbf{Y}$ & \multicolumn{1}{c}{$\mathbf{Z}$} \\
\hline $\mathrm{O}$ & 8 & -0.3428895 & -0.7469401 & 0.0000000 \\
$\mathrm{O}$ & 8 & -1.7121645 & 1.1060193 & 0.0000000 \\
$\mathrm{~N}$ & 7 & -2.5990977 & -0.9772631 & 0.0000000 \\
$\mathrm{C}$ & 6 & 1.9708044 & -1.1637659 & 0.0000000 \\
$\mathrm{C}$ & 6 & 0.9556666 & -0.0210388 & 0.0000000 \\
$\mathrm{C}$ & 6 & 1.0666436 & 0.8209539 & -1.2738815 \\
$\mathrm{C}$ & 6 & 1.0666436 & 0.8209539 & 1.2738815 \\
$\mathrm{C}$ & 6 & -1.5081790 & -0.1007254 & 0.0000000 \\
$\mathrm{H}$ & 1 & 2.9873225 & -0.7574348 & 0.0000000 \\
$\mathrm{H}$ & 1 & 1.8463332 & -1.7910808 & -0.8881016 \\
$\mathrm{H}$ & 1 & 1.8463332 & -1.7910808 & 0.8881016 \\
$\mathrm{H}$ & 1 & 2.0685093 & 1.2610015 & -1.3294038 \\
$\mathrm{H}$ & 1 & 0.3327918 & 1.6291523 & -1.2841589 \\
$\mathrm{H}$ & 1 & 0.9210249 & 0.1946154 & -2.1603947 \\
$\mathrm{H}$ & 1 & 2.0685093 & 1.2610015 & 1.3294038 \\
$\mathrm{H}$ & 1 & 0.9210249 & 0.1946154 & 2.1603947 \\
$\mathrm{H}$ & 1 & 0.3327918 & 1.6291523 & 1.2841589 \\
\hline
\end{tabular}


Table S12. B3LYP/6-31G* calculated IR frequencies $\left(\mathrm{cm}^{-1}\right.$, unscaled) and intensities for syn-triplet $t$-butoxycarbonylnitrene $\mathbf{4} \mathbf{t}_{\text {syn }}$.

\begin{tabular}{lrlr}
\hline Frequency & Intensity & Frequency & Intensity \\
\hline 84 & 0.28 & 1281 & 16.02 \\
105 & 0.10 & 1284 & 163.88 \\
184 & 1.53 & 1300 & 42.34 \\
185 & 0.08 & 1433 & 16.37 \\
234 & 0.01 & 1435 & 14.38 \\
268 & 0.09 & 1459 & 9.33 \\
314 & 1.60 & 1501 & 0.83 \\
330 & 8.80 & 1517 & 0.13 \\
351 & 0.52 & 1519 & 1.43 \\
427 & 0.46 & 1526 & 12.11 \\
456 & 0.76 & 1532 & 1.50 \\
459 & 13.90 & 1552 & 8.36 \\
623 & 1.36 & 1672 & 215.45 \\
681 & 38.13 & 3062 & 18.09 \\
758 & 9.72 & 3062 & 5.73 \\
839 & 18.14 & 3070 & 14.22 \\
935 & 0.13 & 3125 & 7.00 \\
938 & 0.42 & 3127 & 12.28 \\
988 & 0.14 & 3142 & 33.35 \\
1000 & 41.86 & 3143 & 24.14 \\
1069 & 0.50 & 3165 & 1.30 \\
1071 & 4.86 & 3169 & 13.09 \\
1207 & 210.37 & & \\
\hline
\end{tabular}


Table S13. B3LYP/6-31G* optimized geometries, energies, and thermal corrections for anti-singlet t-butoxycarbonylnitrene $\mathbf{4} \boldsymbol{s}_{\text {anti }}$

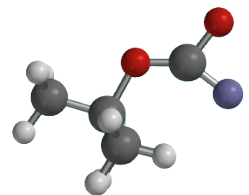

Dipole Moment UB3LYP/6-31G* energy Zero-point correction Thermal correction to energy
5.03 Debye -401.038454 Hartrees 0.138535308 Hartrees 0.147842908 Hartrees

\begin{tabular}{ccrrr}
\hline Atom & $\begin{array}{c}\text { Atomic } \\
\text { Number }\end{array}$ & \multicolumn{3}{c}{ Cartesian Coordinates } \\
& $\mathbf{X}$ & \multicolumn{1}{c}{$\mathbf{Y}$} & \multicolumn{1}{c}{$\mathbf{Z}$} \\
\hline $\mathrm{O}$ & 8 & -0.2534488 & -0.8054490 & 0.0038519 \\
$\mathrm{O}$ & 8 & -2.5802651 & -0.7059322 & 0.0002841 \\
$\mathrm{~N}$ & 7 & -1.9175779 & 0.9822184 & -0.0001882 \\
$\mathrm{C}$ & 6 & 1.0025075 & 0.0070112 & -0.0005382 \\
$\mathrm{C}$ & 6 & 1.0396760 & 0.8597119 & 1.2683846 \\
$\mathrm{C}$ & 6 & 1.0394668 & 0.8454868 & -1.2788467 \\
$\mathrm{C}$ & 6 & 2.0921441 & -1.0613689 & 0.0062179 \\
$\mathrm{C}$ & 6 & -1.4007152 & -0.1848331 & 0.0016673 \\
$\mathrm{H}$ & 1 & 0.9596714 & 0.2278842 & 2.1587200 \\
$\mathrm{H}$ & 1 & 0.2285068 & 1.5942245 & 1.2904215 \\
$\mathrm{H}$ & 1 & 1.9900416 & 1.4018821 & 1.3162592 \\
$\mathrm{H}$ & 1 & 0.2272309 & 1.5783537 & -1.3074743 \\
$\mathrm{H}$ & 1 & 0.9595943 & 0.2035490 & -2.1619311 \\
$\mathrm{H}$ & 1 & 1.9892788 & 1.3879378 & -1.3328696 \\
$\mathrm{H}$ & 1 & 2.0091345 & -1.6912604 & 0.8970262 \\
$\mathrm{H}$ & 1 & 3.0775265 & -0.5847827 & 0.0065926 \\
$\mathrm{H}$ & 1 & 2.0132968 & -1.6983150 & -0.8798245 \\
\hline
\end{tabular}


Table S14. B3LYP/6-31G* calculated IR frequencies $\left(\mathrm{cm}^{-1}\right.$, unscaled) and intensities for anti-singlet $t$-butoxycarbonylnitrene $\mathbf{4} \boldsymbol{s}_{\text {anti }}$.

\begin{tabular}{lrlr}
\hline Frequency & Intensity & Frequency & Intensity \\
\hline 50 & 0.05 & 1285 & 16.66 \\
112 & 0.44 & 1298 & 23.26 \\
168 & 3.10 & 1405 & 75.01 \\
201 & 0.06 & 1432 & 17.96 \\
255 & 0.03 & 1438 & 16.05 \\
268 & 0.16 & 1461 & 8.01 \\
307 & 3.94 & 1501 & 0.63 \\
345 & 0.14 & 1516 & 0.02 \\
359 & 3.08 & 1516 & 0.32 \\
424 & 3.73 & 1525 & 1.77 \\
453 & 0.65 & 1529 & 9.07 \\
469 & 7.03 & 1549 & 5.39 \\
574 & 3.36 & 1810 & 379.44 \\
639 & 29.67 & 3060 & 10.05 \\
754 & 7.75 & 3062 & 7.52 \\
842 & 29.00 & 3069 & 5.46 \\
936 & 0.07 & 3132 & 0.93 \\
944 & 0.24 & 3134 & 7.01 \\
988 & 0.00 & 3138 & 5.00 \\
1056 & 24.75 & 3141 & 23.29 \\
1068 & 1.01 & 3145 & 31.56 \\
1095 & 93.59 & 3146 & 24.24 \\
1213 & 90.75 & & \\
\hline
\end{tabular}


Table S15. B3LYP/6-31G* optimized geometries, energies, and thermal corrections for anti-triplet t-butoxycarbonylnitrene $\mathbf{4} \mathbf{t}_{\text {anti }}$

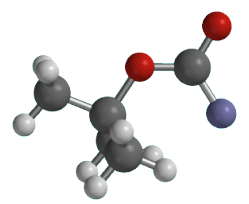

Dipole Moment UB3LYP/6-31G* energy

4.16 Debye Zero-point correction -401.049860 Hartrees Thermal correction to energy 0.137551343 Hartrees 0.146845346 Hartrees

\begin{tabular}{ccrrr}
\hline Atom & $\begin{array}{c}\text { Atomic } \\
\text { Number }\end{array}$ & \multicolumn{3}{c}{ Cartesian Coordinates } \\
& $\mathbf{X}$ & \multicolumn{1}{c}{$\mathbf{Y}$} & \multicolumn{1}{c}{$\mathbf{Z}$} \\
\hline $\mathrm{O}$ & 8 & -0.3325283 & -0.7490762 & 0.0002323 \\
$\mathrm{O}$ & 8 & -2.5680307 & -0.7957221 & -0.0000714 \\
$\mathrm{~N}$ & 7 & -1.6505805 & 1.2338505 & 0.0001032 \\
$\mathrm{C}$ & 6 & 1.9758952 & -1.1619497 & -0.0011845 \\
$\mathrm{C}$ & 6 & 0.9574946 & -0.0205840 & 0.0000005 \\
$\mathrm{C}$ & 6 & 1.0759895 & 0.8211867 & -1.2738821 \\
$\mathrm{C}$ & 6 & 1.0770784 & 0.8195362 & 1.2748557 \\
$\mathrm{C}$ & 6 & -1.5260984 & -0.1470822 & 0.0000536 \\
$\mathrm{H}$ & 1 & 1.8492832 & -1.7886676 & -0.8891079 \\
$\mathrm{H}$ & 1 & 1.8498704 & -1.7896301 & 0.8861259 \\
$\mathrm{H}$ & 1 & 2.9926207 & -0.7561986 & -0.0013079 \\
$\mathrm{H}$ & 1 & 2.0814419 & 1.2512416 & -1.3384585 \\
$\mathrm{H}$ & 1 & 0.3571225 & 1.6465716 & -1.2833189 \\
$\mathrm{H}$ & 1 & 0.9117293 & 0.1999088 & -2.1603208 \\
$\mathrm{H}$ & 1 & 2.0817384 & 1.2516488 & 1.3377632 \\
$\mathrm{H}$ & 1 & 0.9160914 & 0.1965777 & 2.1607175 \\
$\mathrm{H}$ & 1 & 0.3564817 & 1.6433390 & 1.2868393 \\
\hline
\end{tabular}


Table S16. B3LYP/6-31G* calculated IR frequencies $\left(\mathrm{cm}^{-1}\right.$, unscaled) and intensities for anti-triplet $t$-butoxycarbonylnitrene $\mathbf{4 t}_{\text {anti }}$.

\begin{tabular}{lrlr}
\hline Frequency & Intensity & Frequency & Intensity \\
\hline 76 & 0.05 & 1268 & 60.80 \\
92 & 0.02 & 1281 & 16.32 \\
186 & 0.15 & 1299 & 120.53 \\
186 & 0.06 & 1436 & 16.16 \\
233 & 0.04 & 1436 & 11.68 \\
269 & 0.00 & 1462 & 11.01 \\
311 & 2.79 & 1502 & 0.80 \\
351 & 0.53 & 1510 & 0.03 \\
360 & 2.21 & 1515 & 0.41 \\
402 & 3.48 & 1529 & 7.12 \\
457 & 2.31 & 1530 & 1.42 \\
515 & 15.99 & 1545 & 5.22 \\
562 & 5.87 & 1616 & 240.38 \\
659 & 37.40 & 3057 & 11.21 \\
757 & 8.46 & 3060 & 7.44 \\
837 & 15.30 & 3069 & 7.28 \\
933 & 0.10 & 3127 & 0.25 \\
936 & 1.03 & 3130 & 17.56 \\
984 & 0.00 & 3134 & 7.09 \\
1006 & 40.34 & 3136 & 29.06 \\
1064 & 0.17 & 3144 & 33.78 \\
1067 & 1.18 & 3146 & 24.63 \\
1202 & 228.38 & & \\
\hline
\end{tabular}


Table S17. B3LYP/6-31G* optimized geometries, energies, and thermal corrections for the syn-singlet hydroxycarbonylnitrene ${ }^{1} \mathbf{H O C}(\mathbf{O}) \mathbf{N}_{\text {syn }}$

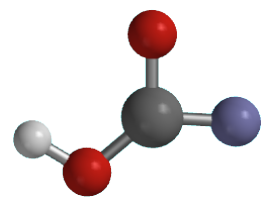

Dipole Moment B3LYP/6-31G* energy Zero-point correction Thermal correction to energy
3.46 Debye -243.773552 Hartrees 0.025526570 Hartrees 0.030307679 Hartrees

\begin{tabular}{ccrrr}
\hline Atom & $\begin{array}{c}\text { Atomic } \\
\text { Number }\end{array}$ & \multicolumn{2}{c}{ Cartesian Coordinates (Angstroms) } \\
& $\mathbf{X}$ & $\mathbf{Y}$ & $\mathbf{Z}$ \\
\hline $\mathrm{O}$ & 8 & 1.3445373 & -0.1534703 & 0.0000000 \\
$\mathrm{O}$ & 8 & -0.7592090 & 0.9264737 & 0.0000000 \\
$\mathrm{~N}$ & 7 & -0.9357988 & -0.9174864 & 0.0000000 \\
$\mathrm{C}$ & 6 & 0.0284869 & -0.0862276 & 0.0000000 \\
$\mathrm{H}$ & 1 & 1.6970436 & 0.7557431 & 0.0000000 \\
\hline
\end{tabular}

Table S18. B3LYP/6-31G* calculated IR frequencies $\left(\mathrm{cm}^{-1}\right.$, unscaled) and intensities for syn-singlet hydroxycarbonylnitrene ${ }^{1} \mathrm{HOC}(0) \mathbf{N}_{\text {syn }}$

\begin{tabular}{lr}
\hline Frequency & Intensity \\
\hline 422 & 11.39 \\
433 & 137.39 \\
490 & 24.40 \\
622 & 33.58 \\
1027 & 47.30 \\
1181 & 94.13 \\
1485 & 142.75 \\
1845 & 227.83 \\
3699 & 100.97 \\
\hline
\end{tabular}


Table S19. B3LYP/6-31G* optimized geometries, energies, and thermal corrections for the syn-triplet hydroxycarbonylnitrene ${ }^{3} \mathrm{HOC}(0) \mathbf{N}_{s y n}$

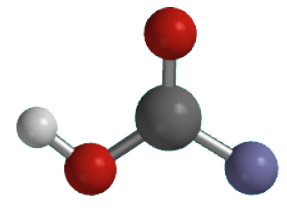

Dipole Moment B3LYP/6-31G* energy Zero-point correction Thermal correction to energy

2.29 Debye
-243.794368 Hartrees
0.025213944 Hartrees
0.029846852 Hartrees

\begin{tabular}{ccrrr}
\hline Atom & $\begin{array}{c}\text { Atomic } \\
\text { Number }\end{array}$ & \multicolumn{2}{c}{ Cartesian Coordinates (Angstroms) } \\
& $\mathbf{X}$ & $\mathbf{Y}$ & $\mathbf{Z}$ \\
\hline $\mathrm{O}$ & 8 & 1.2043522 & -0.3416958 & 0.0000000 \\
$\mathrm{O}$ & 8 & -0.4979783 & 1.1764682 & 0.0000000 \\
$\mathrm{~N}$ & 7 & -0.9798527 & -1.0446951 & 0.0000000 \\
$\mathrm{C}$ & 6 & -0.0882084 & 0.0263958 & 0.0000000 \\
$\mathrm{H}$ & 1 & 1.7372279 & 0.4763113 & 0.0000000 \\
\hline
\end{tabular}

Table S20. B3LYP/6-31G* calculated IR frequencies $\left(\mathrm{cm}^{-1}\right.$, unscaled) and intensities for syn-triplet hydroxycarbonylnitrene ${ }^{3} \mathrm{HOC}(0) \mathbf{N}_{\text {syn }}$

\begin{tabular}{lr}
\hline Frequency & Intensity \\
\hline 428 & 3.56 \\
534 & 54.35 \\
562 & 44.28 \\
701 & 104.83 \\
923 & 1.37 \\
1169 & 257.92 \\
1394 & 29.57 \\
1678 & 208.65 \\
3679 & 76.53 \\
\hline
\end{tabular}


Table S21. B3LYP/6-31G* optimized geometries, energies, and thermal corrections for the anti-singlet hydroxycarbonylnitrene ${ }^{1} \mathbf{H O C}(0) \mathbf{N}_{\text {anti }}$

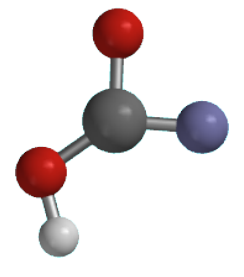

Dipole Moment B3LYP/6-31G* energy Zero-point correction Thermal correction to energy
3.76 Debye -243.772572 Hartrees 0.025567362 Hartrees 0.030325771 Hartrees

\begin{tabular}{ccrrr}
\hline Atom & $\begin{array}{c}\text { Atomic } \\
\text { Number }\end{array}$ & \multicolumn{2}{c}{ Cartesian Coordinates (Angstroms) } \\
& $\mathbf{X}$ & $\mathbf{Y}$ & $\mathbf{Z}$ \\
\hline $\mathrm{O}$ & 8 & 1.3422017 & 0.1459845 & 0.0000000 \\
$\mathrm{O}$ & 8 & -0.8792331 & 0.8454853 & 0.0000000 \\
$\mathrm{~N}$ & 7 & -0.8183908 & -0.9907199 & 0.0000000 \\
$\mathrm{C}$ & 6 & 0.0375852 & -0.0470615 & 0.0000000 \\
$\mathrm{H}$ & 1 & 1.7994753 & -0.7143500 & 0.0000000 \\
\hline
\end{tabular}

Table S22. B3LYP/6-31G* calculated IR frequencies $\left(\mathrm{cm}^{-1}\right.$, unscaled) and intensities for anti-singlet hydroxycarbonylnitrene ${ }^{1} \mathbf{H O C}(\mathbf{0}) \mathbf{N}_{\text {anti }}$

\begin{tabular}{lr}
\hline Frequency & Intensity \\
\hline 434 & 120.71 \\
438 & 21.36 \\
498 & 7.83 \\
625 & 42.12 \\
1022 & 34.80 \\
1179 & 138.76 \\
1501 & 39.67 \\
1818 & 287.42 \\
3706 & 90.39 \\
\hline
\end{tabular}


Table S23. B3LYP/6-31G* optimized geometries, energies, and thermal corrections for the anti-triplet hydroxycarbonylnitrene ${ }^{3} \mathrm{HOC}(0) \mathbf{N}_{a n t i}$

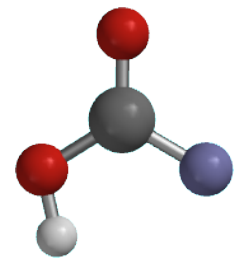

Dipole Moment B3LYP/6-31G* energy Zero-point correction Thermal correction to energy
2.91 Debye
-243.790114 Hartrees
0.024870581 Hartrees
0.029566144 Hartrees

\begin{tabular}{ccrrr}
\hline Atom & $\begin{array}{c}\text { Atomic } \\
\text { Number }\end{array}$ & \multicolumn{2}{c}{ Cartesian Coordinates (Angstroms) } \\
& 8 & 1.1850682 & 0.4237265 & 0.0000000 \\
\hline $\mathrm{O}$ & 8 & -1.0366096 & 0.7940118 & 0.0000000 \\
$\mathrm{O}$ & 7 & -0.3378040 & -1.3540614 & 0.0000000 \\
$\mathrm{~N}$ & 6 & -0.0995243 & 0.0148493 & 0.0000000 \\
$\mathrm{C}$ & 1 & 1.7741055 & -0.3525722 & 0.0000000 \\
$\mathrm{H}$ & & & & \\
\hline
\end{tabular}

Table S24. B3LYP/6-31G* calculated IR frequencies $\left(\mathrm{cm}^{-1}\right.$, unscaled) and intensities for syn-triplet hydroxycarbonylnitrene ${ }^{3} \mathrm{HOC}(0) \mathbf{N}_{\text {syn }}$

\begin{tabular}{lr}
\hline Frequency & Intensity \\
\hline 410 & 25.08 \\
492 & 154.17 \\
571 & 5.96 \\
658 & 17.53 \\
921 & 23.38 \\
1160 & 1.02 \\
1335 & 358.49 \\
1674 & 111.34 \\
3697 & 70.58 \\
\hline
\end{tabular}


Table S25. B3LYP/6-31G* optimized geometries, energies, and thermal corrections for the singlet formylnitrene ${ }^{1} \mathbf{H C}(\mathbf{0}) \mathbf{N}$

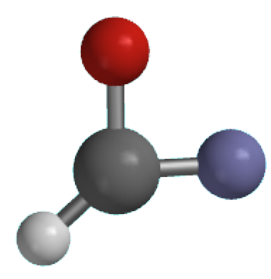

Dipole Moment

B3LYP/6-31G* energy

Zero-point correction

Thermal correction to energy

3.06 Debye

-168.538743 Hartrees

0.020070961 Hartrees

0.024151556 Hartrees

\begin{tabular}{ccrrr}
\hline Atom & $\begin{array}{c}\text { Atomic } \\
\text { Number }\end{array}$ & \multicolumn{2}{c}{ Cartesian Coordinates (Angstroms) } \\
& $\mathbf{X}$ & $\mathbf{Y}$ & $\mathbf{Z}$ \\
\hline $\mathrm{O}$ & 8 & 0.8817724 & 0.2823347 & 0.0000000 \\
$\mathrm{~N}$ & 7 & -0.9003836 & 0.4049873 & 0.0000000 \\
$\mathrm{C}$ & 6 & -0.1052069 & -0.5721507 & 0.0000000 \\
$\mathrm{H}$ & 1 & -0.1202531 & -1.6606852 & 0.0000000 \\
\hline
\end{tabular}

Table S26. B3LYP/6-31G* calculated IR frequencies $\left(\mathrm{cm}^{-1}\right.$, unscaled) and intensities for singlet formylnitrene ${ }^{1} \mathbf{H C}(\mathbf{O}) \mathbf{N}$

\begin{tabular}{lr}
\hline Frequency & Intensity \\
\hline 507 & 11.21 \\
936 & 2.08 \\
1080 & 42.63 \\
1350 & 14.90 \\
1719 & 13.82 \\
3217 & 5.01 \\
\hline
\end{tabular}


Table S27. B3LYP/6-31G* optimized geometries, energies, and thermal corrections for the triplet formylnitrene ${ }^{3} \mathbf{H C}(\mathbf{0}) \mathbf{N}$

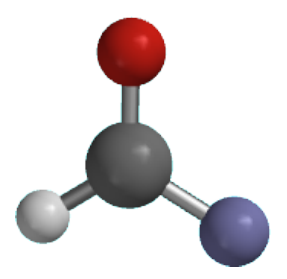

Dipole Moment B3LYP/6-31G* energy Zero-point correction Thermal correction to energy

1.81 Debye -168.551707 Hartrees 0.018805068 Hartrees 0.022925351 Hartrees

\begin{tabular}{ccrrr}
\hline Atom & $\begin{array}{c}\text { Atomic } \\
\text { Number }\end{array}$ & \multicolumn{2}{c}{ Cartesian Coordinates (Angstroms) } \\
& $\mathbf{X}$ & $\mathbf{Y}$ & $\mathbf{Z}$ \\
\hline $\mathrm{O}$ & 8 & 1.0607928 & 0.2492620 & 0.0000000 \\
$\mathrm{~N}$ & 7 & -1.2046797 & 0.2580159 & 0.0000000 \\
$\mathrm{C}$ & 6 & -0.0029283 & -0.3848547 & 0.0000000 \\
$\mathrm{H}$ & 1 & -0.0360151 & -1.4910790 & 0.0000000 \\
\hline
\end{tabular}

Table S28. B3LYP/6-31G* calculated IR frequencies $\left(\mathrm{cm}^{-1}\right.$, unscaled) and intensities for triplet formylnitrene ${ }^{3} \mathrm{HC}(\mathbf{0}) \mathbf{N}$

\begin{tabular}{lr}
\hline Frequency & Intensity \\
\hline 474 & 34.46 \\
900 & 5.48 \\
1034 & 9.38 \\
1380 & 2.84 \\
1474 & 33.12 \\
2992 & 49.76 \\
\hline
\end{tabular}


Table S29. B3LYP/6-31G* optimized geometries, energies, and thermal corrections for the singlet Ethoxycarbonylnitrene rotation transition state $\mathbf{2} \mathbf{s}_{T S}$

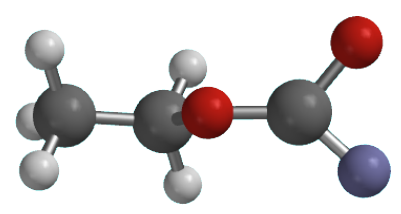

B3LYP/6-31G* energy Zero-point correction Thermal correction to energy
-322.391856 Hartrees

0.082009077 Hartrees

0.088946423 Hartrees

\begin{tabular}{|c|c|c|c|c|}
\hline \multirow[t]{2}{*}{ Atom } & \multirow{2}{*}{$\begin{array}{c}\text { Atomic } \\
\text { Number }\end{array}$} & \multicolumn{3}{|c|}{ Cartesian Coordinates (Angstroms) } \\
\hline & & $\mathbf{X}$ & $\mathbf{Y}$ & $\mathbf{Z}$ \\
\hline 0 & 8 & -0.2022552 & 0.0254922 & 0.5407753 \\
\hline 0 & 8 & 1.8880580 & 0.8949285 & -0.1414016 \\
\hline $\mathrm{N}$ & 7 & 1.9374485 & -0.9439754 & -0.0370750 \\
\hline $\mathrm{C}$ & 6 & -2.5630977 & 0.0670042 & 0.1159209 \\
\hline $\mathrm{C}$ & 6 & -1.2027368 & -0.1222912 & -0.5194385 \\
\hline $\mathrm{C}$ & 6 & 1.0604425 & -0.0465543 & 0.1541921 \\
\hline $\mathrm{H}$ & 1 & -1.0015728 & 0.6325078 & -1.2870332 \\
\hline $\mathrm{H}$ & 1 & -1.0903275 & -1.1182631 & -0.9604677 \\
\hline $\mathrm{H}$ & 1 & -3.3401540 & -0.0355668 & -0.6490800 \\
\hline $\mathrm{H}$ & 1 & -2.6461325 & 1.0610433 & 0.5651179 \\
\hline $\mathrm{H}$ & 1 & -2.7380235 & -0.6842104 & 0.8919511 \\
\hline
\end{tabular}

Table S30. B3LYP/6-31G* calculated IR frequencies $\left(\mathrm{cm}^{-1}\right.$, unscaled) and intensities for singlet Ethoxycarbonylnitrene rotation transition state $\mathbf{2} \mathbf{s}_{T S}$

\begin{tabular}{lrlr}
\hline Frequency & Intensity & Frequency & Intensity \\
\hline-127 & 1.15 & 1307 & 2.12 \\
29 & 0.72 & 1375 & 115.17 \\
172 & 4.58 & 1415 & 13.59 \\
262 & 0.14 & 1447 & 29.74 \\
335 & 14.39 & 1512 & 7.16 \\
438 & 4.46 & 1529 & 2.99 \\
507 & 6.72 & 1545 & 6.49 \\
687 & 4.42 & 1804 & 313.78 \\
824 & 1.28 & 3067 & 20.77 \\
845 & 24.93 & 3067 & 8.45 \\
1014 & 6.81 & 3114 & 10.16 \\
1087 & 237.53 & 3140 & 17.18 \\
1141 & 7.51 & 3151 & 24.79 \\
1183 & 4.42 & & \\
\hline
\end{tabular}


Table S31. B3LYP/6-31G* optimized geometries, energies, and thermal corrections for the triplet Ethoxycarbonylnitrene rotation transition state $\mathbf{2 t}_{T S}$

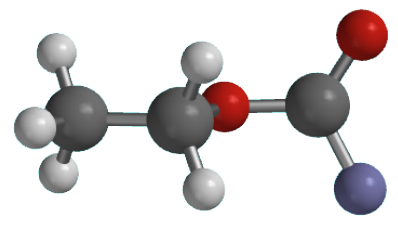

B3LYP/6-31G* energy Zero-point correction Thermal correction to energy
-322.406497 Hartrees

0.081190566 Hartrees

0.088100374 Hartrees

\begin{tabular}{|c|c|c|c|c|}
\hline \multirow[t]{2}{*}{ Atom } & \multirow{2}{*}{$\begin{array}{c}\text { Atomic } \\
\text { Number }\end{array}$} & \multicolumn{3}{|c|}{ Cartesian Coordinates (Angstroms) } \\
\hline & & $\mathbf{X}$ & $\mathbf{Y}$ & $\mathbf{Z}$ \\
\hline 0 & 8 & -0.1365246 & -0.0311651 & 0.5132095 \\
\hline 0 & 8 & 1.8242503 & -1.0294335 & -0.0860061 \\
\hline $\mathrm{N}$ & 7 & 1.7171471 & 1.2320259 & -0.0495643 \\
\hline $\mathrm{C}$ & 6 & -2.5041736 & -0.0228614 & 0.1464102 \\
\hline $\mathrm{C}$ & 6 & -1.1481864 & 0.0205148 & -0.5286150 \\
\hline $\mathrm{C}$ & 6 & 1.1585924 & -0.0213163 & 0.0985252 \\
\hline $\mathrm{H}$ & 1 & -2.6340254 & 0.8375033 & 0.8099689 \\
\hline $\mathrm{H}$ & 1 & -2.6103459 & -0.9382758 & 0.7361532 \\
\hline $\mathrm{H}$ & 1 & -3.2949606 & -0.0012761 & -0.6112882 \\
\hline $\mathrm{H}$ & 1 & -1.0184863 & 0.9428507 & -1.1085146 \\
\hline $\mathrm{H}$ & 1 & -1.0014113 & -0.8382181 & -1.1949186 \\
\hline
\end{tabular}

Table S32. B3LYP/6-31G* calculated IR frequencies $\left(\mathrm{cm}^{-1}\right.$, unscaled) and intensities for triplet Ethoxycarbonylnitrene rotation transition state $\mathbf{2} \mathbf{t}_{T S}$

\begin{tabular}{lrlr}
\hline Frequency & Intensity & Frequency & Intensity \\
\hline-122 & 2.47 & 1200 & 309.77 \\
63 & 0.26 & 1312 & 1.25 \\
187 & 2.60 & 1417 & 19.62 \\
246 & 0.28 & 1450 & 32.76 \\
335 & 8.94 & 1512 & 6.43 \\
436 & 1.65 & 1530 & 2.42 \\
579 & 15.80 & 1548 & 8.55 \\
693 & 13.28 & 1610 & 143.90 \\
832 & 2.56 & 3046 & 26.63 \\
839 & 20.77 & 3067 & 11.01 \\
975 & 18.16 & 3092 & 18.84 \\
1061 & 116.29 & 3139 & 19.88 \\
1138 & 26.15 & 3149 & 23.25 \\
1185 & 8.75 & & \\
\hline
\end{tabular}


Table S33. B3LYP/6-31G* optimized geometries, energies, and thermal corrections for the singlet $t$-butoxycarbonylnitrene rotation transition state $\mathbf{4} \mathbf{s}_{T S}$

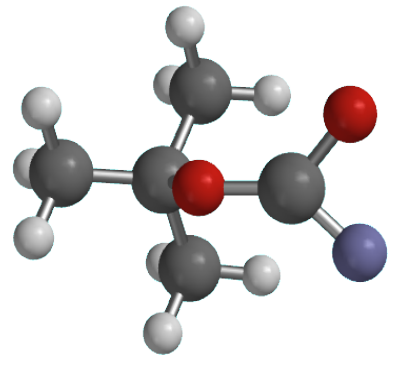

B3LYP/6-31G* energy Zero-point correction Thermal correction to energy
-401.029715 Hartrees

0.137859437 Hartrees

0.147244089 Hartrees

\begin{tabular}{ccrrr}
\hline Atom & $\begin{array}{c}\text { Atomic } \\
\text { Number }\end{array}$ & \multicolumn{2}{c}{ Cartesian Coordinates } & \multicolumn{1}{c}{ X Angstroms) } \\
& 8 & 0.2061566 & -0.0025986 & 0.8602736 \\
\hline $\mathrm{O}$ & 8 & 2.2221037 & 0.8748164 & -0.0167456 \\
$\mathrm{O}$ & 7 & 2.2390164 & -0.9566874 & -0.0362335 \\
$\mathrm{~N}$ & 6 & -0.9927710 & 0.0012238 & -0.0429245 \\
$\mathrm{C}$ & 6 & -1.0650218 & -1.3538514 & -0.7452097 \\
$\mathrm{C}$ & 6 & -0.8640247 & 1.1630065 & -1.0277721 \\
$\mathrm{C}$ & 6 & -2.1470461 & 0.2030776 & 0.9324694 \\
$\mathrm{C}$ & 6 & 1.4029820 & -0.0677014 & 0.3162345 \\
$\mathrm{C}$ & 1 & -1.1397269 & -2.1647983 & -0.0139120 \\
$\mathrm{H}$ & 1 & -0.1810307 & -1.5255437 & -1.3677193 \\
$\mathrm{H}$ & 1 & -1.9479560 & -1.3887423 & -1.3925644 \\
$\mathrm{H}$ & 1 & -0.0127745 & 1.0295064 & -1.7028397 \\
$\mathrm{H}$ & 1 & -0.7363814 & 2.1096966 & -0.4943243 \\
$\mathrm{H}$ & 1 & -1.7721562 & 1.2275101 & -1.6368100 \\
$\mathrm{H}$ & 1 & -2.1738032 & -0.6063582 & 1.6679653 \\
$\mathrm{H}$ & 1 & -3.0957684 & 0.2087446 & 0.3860131 \\
$\mathrm{H}$ & 1 & -2.0443103 & 1.1545234 & 1.4628163 \\
$\mathrm{H}$ & & & & \\
\hline
\end{tabular}


Table S34. B3LYP/6-31G* calculated IR frequencies $\left(\mathrm{cm}^{-1}\right.$, unscaled) and intensities for singlet $t$-butoxycarbonylnitrene rotation transition state $4 \mathbf{s}_{T S}$

\begin{tabular}{lrlr}
\hline Frequency & Intensity & Frequency & Intensity \\
\hline-74 & 0.09 & 1284 & 13.95 \\
52 & 0.08 & 1299 & 23.24 \\
158 & 5.00 & 1363 & 87.86 \\
206 & 0.04 & 1435 & 14.92 \\
260 & 0.04 & 1437 & 13.95 \\
270 & 0.08 & 1461 & 9.23 \\
301 & 6.39 & 1503 & 0.28 \\
322 & 0.58 & 1509 & 0.41 \\
376 & 6.02 & 1513 & 0.08 \\
422 & 3.09 & 1528 & 2.71 \\
427 & 3.88 & 1532 & 6.24 \\
463 & 0.47 & 1542 & 6.67 \\
528 & 10.14 & 1799 & 311.22 \\
637 & 7.85 & 3059 & 9.21 \\
767 & 16.15 & 3062 & 8.61 \\
836 & 47.61 & 3069 & 6.62 \\
933 & 0.03 & 3130 & 0.32 \\
939 & 0.58 & 3134 & 7.89 \\
985 & 0.01 & 3139 & 10.30 \\
1049 & 103.11 & 3141 & 22.88 \\
1066 & 0.85 & 3145 & 25.02 \\
1078 & 69.95 & 3147 & 24.16 \\
1207 & 96.44 & & \\
\hline
\end{tabular}


Table S35. B3LYP/6-31G* optimized geometries, energies, and thermal corrections for the triplet $t$-butoxycarbonylnitrene rotation transition state $\mathbf{4} \mathbf{t}_{T S}$

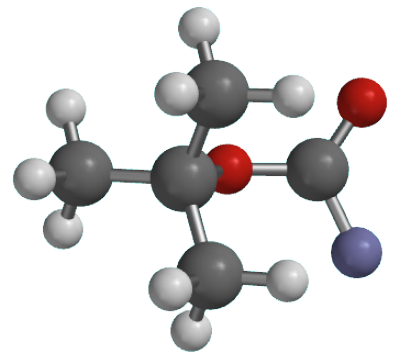

B3LYP/6-31G* energy Zero-point correction Thermal correction to energy
-401.042161 Hartrees

0.136692230 Hartrees

0.146075625 Hartrees

\begin{tabular}{ccrrr}
\hline Atom & $\begin{array}{c}\text { Atomic } \\
\text { Number }\end{array}$ & \multicolumn{2}{c}{ Cartesian Coordinates } & \multicolumn{1}{c}{ X Angstroms) } \\
& 8 & 0.2779540 & -0.0905718 & 0.7973229 \\
\hline O & 8 & 2.2010301 & -1.0026846 & -0.0118119 \\
$\mathrm{O}$ & 7 & 1.9970753 & 1.2450350 & -0.0052340 \\
$\mathrm{~N}$ & 6 & -0.9517879 & -1.1819694 & -1.0150273 \\
$\mathrm{C}$ & 6 & -0.9493680 & -0.0093622 & -0.0316406 \\
$\mathrm{C}$ & 6 & -2.0692200 & -0.1382267 & 0.9988137 \\
$\mathrm{C}$ & 6 & -1.0026430 & 1.3419064 & -0.7485921 \\
$\mathrm{C}$ & 6 & 1.5037401 & -0.0248348 & 0.2267034 \\
$\mathrm{C}$ & 1 & -1.8911628 & -1.1916496 & -1.5788074 \\
$\mathrm{H}$ & 1 & -0.8536890 & -2.1317352 & -0.4816151 \\
$\mathrm{H}$ & 1 & -0.1289065 & -1.1064467 & -1.7334256 \\
$\mathrm{H}$ & 1 & -3.0434602 & -0.1056514 & 0.4998173 \\
$\mathrm{H}$ & 1 & -2.0221535 & 0.6803986 & 1.7237292 \\
$\mathrm{H}$ & 1 & -1.9857141 & -1.0855953 & 1.5400783 \\
$\mathrm{H}$ & 1 & -1.9497468 & 1.4354780 & -1.2911735 \\
$\mathrm{H}$ & 1 & -0.1906521 & 1.4464267 & -1.4766552 \\
$\mathrm{H}$ & 1 & -0.9302420 & 2.1645007 & -0.0309412 \\
$\mathrm{H}$ & & & & \\
\hline
\end{tabular}


Table S36. B3LYP/6-31G* calculated IR frequencies $\left(\mathrm{cm}^{-1}\right.$, unscaled) and intensities for triplet $t$-butoxycarbonylnitrene rotation transition state $\mathbf{4 t}_{T S}$

\begin{tabular}{lrlr}
\hline Frequency & Intensity & Frequency & Intensity \\
\hline-66 & 1.27 & 1234 & 26.58 \\
37 & 0.29 & 1278 & 16.22 \\
165 & 1.86 & 1295 & 20.05 \\
198 & 0.04 & 1424 & 15.80 \\
254 & 0.01 & 1431 & 13.09 \\
264 & 0.06 & 1455 & 7.99 \\
296 & 2.74 & 1500 & 0.21 \\
318 & 1.58 & 1507 & 0.47 \\
380 & 2.45 & 1513 & 0.07 \\
404 & 0.56 & 1523 & 3.93 \\
440 & 5.70 & 1528 & 4.50 \\
476 & 0.99 & 1542 & 4.76 \\
570 & 16.58 & 1601 & 165.48 \\
667 & 7.62 & 3056 & 8.38 \\
756 & 23.09 & 3059 & 15.03 \\
834 & 30.34 & 3066 & 4.75 \\
927 & 0.12 & 3122 & 4.86 \\
935 & 3.02 & 3128 & 28.68 \\
978 & 2.28 & 3135 & 18.22 \\
983 & 87.39 & 3140 & 3.48 \\
1055 & 1.25 & 3142 & 27.47 \\
1066 & 2.01 & 3146 & 27.15 \\
1176 & 346.59 & & \\
\hline
\end{tabular}


Table S37. B3LYP/6-31G* optimized geometries, energies, and thermal corrections for the syn-ethoxycarbonylnitrene-acetonitrile ylide $\mathbf{8}$.

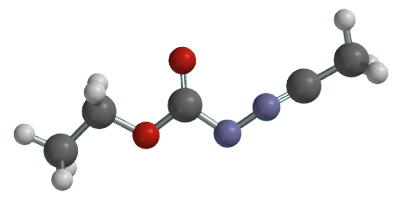

B3LYP/6-31G* energy

Zero-point correction

Thermal correction to energy
-455.230639 Hartrees

0.133213464 Hartrees

0.142894936 Hartrees

\begin{tabular}{ccrrr}
\hline Atom & $\begin{array}{c}\text { Atomic } \\
\text { Number }\end{array}$ & \multicolumn{2}{c}{ Cartesian Coordinates } \\
$\mathbf{X}$ & $\mathbf{Y}$ & \multicolumn{1}{c}{ (Angstroms) } \\
\hline $\mathrm{C}$ & 6 & 0.3523481 & -0.0898696 & 0.0000000 \\
$\mathrm{O}$ & 8 & 1.5868383 & 0.4585713 & 0.0000000 \\
$\mathrm{C}$ & 6 & 2.6726596 & -0.4866861 & 0.0000000 \\
$\mathrm{H}$ & 1 & 2.5890821 & -1.1301190 & 0.8826815 \\
$\mathrm{H}$ & 1 & 2.5890821 & -1.1301190 & -0.8826815 \\
$\mathrm{C}$ & 6 & 3.9644716 & 0.3098778 & 0.0000000 \\
$\mathrm{H}$ & 1 & 4.0289178 & 0.9483600 & 0.8870024 \\
$\mathrm{H}$ & 1 & 4.0289178 & 0.9483600 & -0.8870024 \\
$\mathrm{H}$ & 1 & 4.8230558 & -0.3708331 & 0.0000000 \\
$\mathrm{O}$ & 8 & 0.1296893 & -1.2899111 & 0.0000000 \\
$\mathrm{~N}$ & 7 & -0.5819369 & 0.9453118 & 0.0000000 \\
$\mathrm{~N}$ & 7 & -1.7793356 & 0.4945702 & 0.0000000 \\
$\mathrm{C}$ & 6 & -2.8975220 & 0.1811652 & 0.0000000 \\
$\mathrm{C}$ & 6 & -4.3015387 & -0.2053898 & 0.0000000 \\
$\mathrm{H}$ & 1 & -4.8143333 & 0.1747462 & 0.8908718 \\
$\mathrm{H}$ & 1 & -4.3762147 & -1.2981820 & 0.0000000 \\
$\mathrm{H}$ & 1 & -4.8143333 & 0.1747462 & -0.8908718 \\
\hline
\end{tabular}


Table S38. B3LYP/6-31G* calculated IR frequencies $\left(\mathrm{cm}^{-1}\right.$, unscaled) and intensities for the syn-ethoxycarbonylnitrene-acetonitrile ylide $\mathbf{8}$.

\begin{tabular}{lrlr}
\hline Frequency & Intensity & Frequency & Intensity \\
\hline 25 & 2.04 & 1193 & 4.17 \\
31 & 0.20 & 1288 & 1151.09 \\
74 & 7.45 & 1301 & 0.28 \\
78 & 6.13 & 1335 & 600.69 \\
116 & 0.09 & 1416 & 51.98 \\
168 & 0.36 & 1444 & 19.77 \\
188 & 3.27 & 1452 & 7.87 \\
269 & 1.53 & 1493 & 10.56 \\
274 & 0.41 & 1502 & 9.63 \\
363 & 13.08 & 1517 & 4.62 \\
449 & 2.46 & 1527 & 1.71 \\
491 & 3.86 & 1547 & 4.81 \\
491 & 1.15 & 1768 & 255.51 \\
755 & 24.17 & 2441 & 359.24 \\
763 & 0.56 & 3050 & 15.11 \\
818 & 0.03 & 3059 & 20.59 \\
825 & 16.67 & 3064 & 22.19 \\
920 & 7.11 & 3101 & 13.49 \\
1004 & 11.92 & 3116 & 2.69 \\
1060 & 2.12 & 3128 & 2.70 \\
1060 & 11.19 & 3130 & 29.91 \\
1092 & 96.15 & 3139 & 40.28 \\
1150 & 8.31 & & \\
\hline
\end{tabular}


Table S39. B3LYP/6-31G* optimized geometries, energies, and thermal corrections for the anti-ethoxycarbonylnitrene-acetonitrile ylide $\mathbf{8}$.

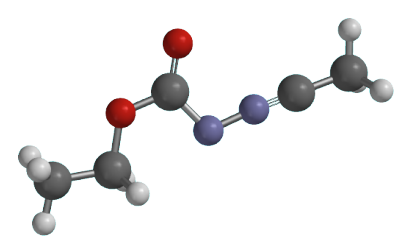

B3LYP/6-31G* energy Zero-point correction Thermal correction to energy
-455.226370 Hartrees

0.133374386 Hartrees

0.143041193 Hartrees

\begin{tabular}{ccrrr}
\hline Atom & $\begin{array}{c}\text { Atomic } \\
\text { Number }\end{array}$ & \multicolumn{2}{c}{ Cartesian Coordinates } & X Angstroms) \\
& 6 & -0.3551402 & -0.6890410 & 0.0006956 \\
\hline $\mathrm{C}$ & 8 & -1.7021155 & -0.5839471 & 0.0008247 \\
$\mathrm{O}$ & 6 & -2.3325364 & 0.7120337 & 0.0025093 \\
$\mathrm{C}$ & 1 & -2.0172880 & 1.2714107 & 0.8898792 \\
$\mathrm{H}$ & 1 & -2.0106817 & 1.2769062 & -0.8788564 \\
$\mathrm{H}$ & 6 & -3.8325710 & 0.4740399 & -0.0030553 \\
$\mathrm{C}$ & 1 & -4.1379832 & -0.0963266 & 0.8799342 \\
$\mathrm{H}$ & 1 & -4.1333520 & -0.0861660 & -0.8940882 \\
$\mathrm{H}$ & 1 & -4.3641576 & 1.4323226 & 0.0010826 \\
$\mathrm{H}$ & 8 & 0.1824679 & -1.7787926 & -0.0001094 \\
$\mathrm{O}$ & 7 & 0.2865589 & 0.5588257 & 0.0010641 \\
$\mathrm{~N}$ & 7 & 1.5591100 & 0.4274010 & 0.0002814 \\
$\mathrm{~N}$ & 6 & 2.7186744 & 0.3819762 & -0.0011667 \\
$\mathrm{C}$ & 6 & 4.1739302 & 0.3327050 & -0.0007562 \\
$\mathrm{C}$ & 1 & 4.5840640 & 0.8280349 & -0.8880230 \\
$\mathrm{H}$ & 1 & 4.4993995 & -0.7131845 & -0.0083984 \\
$\mathrm{H}$ & 1 & 4.5833559 & 0.8150507 & 0.8939689 \\
$\mathrm{H}$ & & & & \\
\hline
\end{tabular}


Table S40. B3LYP/6-31G* calculated IR frequencies $\left(\mathrm{cm}^{-1}\right.$, unscaled) and intensities for the anti-ethoxycarbonylnitrene-acetonitrile ylide $\mathbf{8}$.

\begin{tabular}{lrlr}
\hline Frequency & Intensity & Frequency & Intensity \\
\hline 49 & 2.41 & 1190 & 3.87 \\
71 & 0.59 & 1272 & 1133.02 \\
81 & 6.80 & 1301 & 0.11 \\
88 & 2.75 & 1324 & 405.94 \\
129 & 0.00 & 1407 & 33.02 \\
169 & 0.29 & 1443 & 15.39 \\
188 & 0.24 & 1448 & 2.67 \\
269 & 1.53 & 1494 & 11.08 \\
286 & 0.44 & 1502 & 9.74 \\
335 & 0.84 & 1518 & 4.55 \\
486 & 2.55 & 1529 & 4.92 \\
497 & 3.19 & 1548 & 0.60 \\
544 & 14.67 & 1797 & 368.23 \\
658 & 1.82 & 2445 & 339.15 \\
745 & 25.61 & 3052 & 12.61 \\
823 & 0.22 & 3060 & 24.42 \\
860 & 8.19 & 3062 & 19.92 \\
895 & 0.19 & 3103 & 15.01 \\
996 & 9.60 & 3119 & 2.31 \\
1060 & 11.35 & 3130 & 2.05 \\
1061 & 2.29 & 3131 & 29.00 \\
1089 & 57.08 & 3141 & 38.72 \\
1149 & 90.43 & & \\
\hline
\end{tabular}


Table S41. B3LYP/6-31G* optimized geometries, energies, and thermal corrections for syn-5-ethoxycarbonyliminodibenzothiophene 6 .

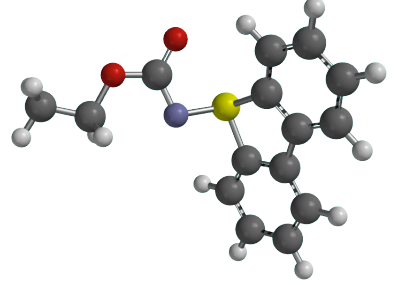

B3LYP/6-31G* energy Zero-point correction Thermal correction to energy
-1182.779443 Hartrees

0.248654539 Hartrees

0.263385183 Hartrees

\begin{tabular}{|c|c|c|c|c|}
\hline \multirow[t]{2}{*}{ Atom } & \multirow{2}{*}{$\begin{array}{c}\text { Atomic } \\
\text { Number }\end{array}$} & \multicolumn{3}{|c|}{ Cartesian Coordinates (Angstroms) } \\
\hline & & $\mathbf{X}$ & $\mathbf{Y}$ & $\mathbf{Z}$ \\
\hline $\mathrm{H}$ & 1 & 0.8073731 & -2.6454561 & -0.7004214 \\
\hline $\mathrm{C}$ & 6 & -0.2102720 & -2.5018047 & -0.3537613 \\
\hline $\mathrm{C}$ & 6 & -2.8505430 & -2.0460356 & 0.5547557 \\
\hline $\mathrm{C}$ & 6 & -0.7515278 & -1.2241001 & -0.2972131 \\
\hline $\mathrm{C}$ & 6 & -1.0126904 & -3.5654987 & 0.0706302 \\
\hline $\mathrm{C}$ & 6 & -2.3199920 & -3.3368979 & 0.5142633 \\
\hline $\mathrm{C}$ & 6 & -2.0577178 & -0.9673578 & 0.1525418 \\
\hline $\mathrm{H}$ & 1 & -0.6162677 & -4.5765617 & 0.0523204 \\
\hline $\mathrm{H}$ & 1 & -2.9331401 & -4.1752833 & 0.8328485 \\
\hline $\mathrm{H}$ & 1 & -3.8683512 & -1.8844373 & 0.8991991 \\
\hline $\mathrm{C}$ & 6 & -2.3889964 & 0.4637118 & 0.1332233 \\
\hline $\mathrm{C}$ & 6 & -2.5767061 & 3.2583145 & 0.0260297 \\
\hline $\mathrm{C}$ & 6 & -1.3273555 & 1.2569649 & -0.3311952 \\
\hline $\mathrm{C}$ & 6 & -3.5703345 & 1.0969473 & 0.5313870 \\
\hline $\mathrm{C}$ & 6 & -3.6556162 & 2.4886373 & 0.4747061 \\
\hline $\mathrm{C}$ & 6 & -1.3938895 & 2.6415070 & -0.3901477 \\
\hline $\mathrm{H}$ & 1 & -4.4116543 & 0.5126600 & 0.8931832 \\
\hline $\mathrm{H}$ & 1 & -4.5713032 & 2.9805116 & 0.7904748 \\
\hline $\mathrm{H}$ & 1 & -0.5434761 & 3.2233263 & -0.7303921 \\
\hline $\mathrm{H}$ & 1 & -2.6554613 & 4.3410816 & 0.0018010 \\
\hline S & 16 & 0.0700215 & 0.2814714 & -0.8829485 \\
\hline $\mathrm{N}$ & 7 & 1.2777348 & 0.8356163 & 0.1121213 \\
\hline $\mathrm{C}$ & 6 & 2.4164788 & 0.1552327 & -0.2199942 \\
\hline 0 & 8 & 2.5099072 & -0.8437529 & -0.9480833 \\
\hline 0 & 8 & 3.4889718 & 0.7115747 & 0.3938449 \\
\hline $\mathrm{C}$ & 6 & 4.7384205 & 0.0278791 & 0.1968831 \\
\hline $\mathrm{H}$ & 1 & 4.6346302 & -1.0155328 & 0.5147167 \\
\hline $\mathrm{H}$ & 1 & 4.9851180 & 0.0210713 & -0.8705651 \\
\hline $\mathrm{C}$ & 6 & 5.7865480 & 0.7648968 & 1.0115466 \\
\hline $\mathrm{H}$ & 1 & 5.5211418 & 0.7694088 & 2.0737419 \\
\hline $\mathrm{H}$ & 1 & 5.8802041 & 1.8035163 & 0.6779616 \\
\hline $\mathrm{H}$ & 1 & 6.7608302 & 0.2758866 & 0.8994338 \\
\hline
\end{tabular}


Table S42. B3LYP/6-31G* calculated IR frequencies $\left(\mathrm{cm}^{-1}\right.$, unscaled) and intensities for syn-5-ethoxycarbonyliminodibenzothiophene 6.

\begin{tabular}{|c|c|c|c|}
\hline Frequency & Intensity & Frequency & Intensity \\
\hline 35 & 0.61 & 1049 & 1.10 \\
\hline 55 & 0.47 & 1057 & 2.21 \\
\hline 61 & 0.07 & 1074 & 3.55 \\
\hline 89 & 1.32 & 1094 & 4.23 \\
\hline 95 & 1.95 & 1127 & 88.14 \\
\hline 119 & 3.92 & 1148 & 8.42 \\
\hline 132 & 1.73 & 1151 & 6.90 \\
\hline 160 & 5.05 & 1160 & 0.87 \\
\hline 185 & 2.37 & 1196 & 2.75 \\
\hline 199 & 0.86 & 1196 & 1.55 \\
\hline 234 & 10.18 & 1198 & 0.64 \\
\hline 285 & 1.44 & 1259 & 3.19 \\
\hline 286 & 0.12 & 1308 & 56.40 \\
\hline 302 & 11.47 & 1309 & 7.78 \\
\hline 374 & 13.23 & 1321 & 1268.37 \\
\hline 400 & 0.76 & 1335 & 49.70 \\
\hline 416 & 0.58 & 1350 & 2.35 \\
\hline 421 & 3.38 & 1384 & 0.27 \\
\hline 443 & 5.91 & 1422 & 91.81 \\
\hline 480 & 3.44 & 1452 & 19.67 \\
\hline 497 & 8.67 & 1475 & 14.76 \\
\hline 509 & 21.53 & 1493 & 26.92 \\
\hline 548 & 12.67 & 1511 & 10.47 \\
\hline 569 & 0.36 & 1518 & 4.41 \\
\hline 629 & 5.33 & 1523 & 1.29 \\
\hline 700 & 1.10 & 1528 & 2.22 \\
\hline 711 & 0.64 & 1550 & 4.44 \\
\hline 726 & 11.92 & 1629 & 2.66 \\
\hline 740 & 1.87 & 1637 & 0.26 \\
\hline 759 & 2.09 & 1645 & 0.50 \\
\hline 769 & 88.09 & 1652 & 0.32 \\
\hline 772 & 69.16 & 1675 & 201.94 \\
\hline 777 & 15.70 & 3058 & 23.38 \\
\hline 792 & 3.90 & 3062 & 25.99 \\
\hline 809 & 29.08 & 3099 & 14.92 \\
\hline 824 & 1.76 & 3129 & 31.91 \\
\hline 882 & 0.03 & 3138 & 40.39 \\
\hline 894 & 0.09 & 3190 & 3.99 \\
\hline 911 & 4.75 & 3193 & 0.69 \\
\hline 950 & 0.71 & 3203 & 4.93 \\
\hline 968 & 1.41 & 3205 & 13.43 \\
\hline 992 & 0.06 & 3214 & 21.26 \\
\hline 1003 & 0.67 & 3215 & 19.72 \\
\hline 1018 & 0.02 & 3226 & 8.78 \\
\hline 1031 & 24.90 & 3237 & 7.00 \\
\hline
\end{tabular}


Table S43. B3LYP/6-31G* optimized geometries, energies, and thermal corrections for anti-5-ethoxycarbonyliminodibenzothiophene 6.

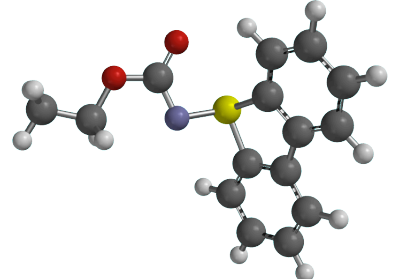

B3LYP/6-31G* energy Zero-point correction Thermal correction to energy
-1182.776940 Hartrees

0.248537685 Hartrees

0.263284060 Hartrees

\begin{tabular}{|c|c|c|c|c|}
\hline \multirow[t]{2}{*}{ Atom } & \multirow{2}{*}{$\begin{array}{c}\text { Atomic } \\
\text { Number }\end{array}$} & \multicolumn{3}{|c|}{ Cartesian Coordinates (Angstroms) } \\
\hline & & $\mathbf{X}$ & $\mathbf{Y}$ & $\mathbf{Z}$ \\
\hline $\mathrm{H}$ & 1 & 0.2843726 & -2.9759812 & -0.6885767 \\
\hline $\mathrm{C}$ & 6 & -0.6517286 & -2.6058961 & -0.2853915 \\
\hline $\mathrm{C}$ & 6 & -3.0608709 & -1.5590988 & 0.7693662 \\
\hline $\mathrm{C}$ & 6 & -0.9171112 & -1.2424965 & -0.2840770 \\
\hline $\mathrm{C}$ & 6 & -1.6163534 & -3.4527635 & 0.2696223 \\
\hline $\mathrm{C}$ & 6 & -2.8085394 & -2.9322823 & 0.7849056 \\
\hline $\mathrm{C}$ & 6 & -2.1013946 & -0.6936168 & 0.2364920 \\
\hline $\mathrm{H}$ & 1 & -1.4365621 & -4.5234690 & 0.2980344 \\
\hline $\mathrm{H}$ & 1 & -3.5507694 & -3.6054427 & 1.2048126 \\
\hline $\mathrm{H}$ & 1 & -3.9920424 & -1.1699972 & 1.1716519 \\
\hline $\mathrm{C}$ & 6 & -2.1354485 & 0.7714462 & 0.1381629 \\
\hline $\mathrm{C}$ & 6 & -1.7686110 & 3.5335086 & -0.1700769 \\
\hline $\mathrm{C}$ & 6 & -0.9752767 & 1.2996306 & -0.4511379 \\
\hline $\mathrm{C}$ & 6 & -3.1304249 & 1.6569443 & 0.5637439 \\
\hline $\mathrm{C}$ & 6 & -2.9397018 & 3.0304297 & 0.4071306 \\
\hline $\mathrm{C}$ & 6 & -0.7703216 & 2.6623955 & -0.6142062 \\
\hline $\mathrm{H}$ & 1 & -4.0415184 & 1.2812181 & 1.0207286 \\
\hline $\mathrm{H}$ & 1 & -3.7110688 & 3.7179165 & 0.7424031 \\
\hline $\mathrm{H}$ & 1 & 0.1464930 & 3.0363122 & -1.0585823 \\
\hline $\mathrm{H}$ & 1 & -1.6326009 & 4.6057047 & -0.2752986 \\
\hline S & 16 & 0.1485134 & 0.0236353 & -1.0192149 \\
\hline $\mathrm{N}$ & 7 & 1.5103730 & 0.3465965 & -0.1163687 \\
\hline $\mathrm{C}$ & 6 & 2.4369512 & -0.5982204 & -0.4700490 \\
\hline 0 & 8 & 2.2390426 & -1.5978089 & -1.1696569 \\
\hline 0 & 8 & 3.6777104 & -0.3981752 & 0.0373888 \\
\hline $\mathrm{C}$ & 6 & 3.9355996 & 0.7745118 & 0.8304317 \\
\hline $\mathrm{H}$ & 1 & 3.7139717 & 1.6712180 & 0.2406752 \\
\hline $\mathrm{H}$ & 1 & 3.2713044 & 0.7797609 & 1.7014835 \\
\hline $\mathrm{C}$ & 6 & 5.3974728 & 0.7235619 & 1.2398866 \\
\hline $\mathrm{H}$ & 1 & 6.0472179 & 0.7167791 & 0.3587960 \\
\hline $\mathrm{H}$ & 1 & 5.6051702 & -0.1778701 & 1.8254138 \\
\hline $\mathrm{H}$ & 1 & 5.6477365 & 1.5990582 & 1.8498036 \\
\hline
\end{tabular}


Table S44. B3LYP/6-31G* calculated IR frequencies $\left(\mathrm{cm}^{-1}\right.$, unscaled) and intensities for anti-5-ethoxycarbonyliminodibenzothiophene $\mathbf{6}$.

\begin{tabular}{|c|c|c|c|}
\hline Frequency & Intensity & Frequency & Intensity \\
\hline 27 & 0.73 & 1049 & 1.79 \\
\hline 51 & 0.99 & 1057 & 1.71 \\
\hline 60 & 0.35 & 1073 & 4.85 \\
\hline 72 & 4.77 & 1094 & 5.49 \\
\hline 96 & 1.27 & 1116 & 52.22 \\
\hline 127 & 2.05 & 1148 & 73.69 \\
\hline 151 & 0.48 & 1152 & 3.95 \\
\hline 158 & 4.89 & 1160 & 0.62 \\
\hline 184 & 7.69 & 1191 & 3.25 \\
\hline 202 & 1.68 & 1197 & 0.43 \\
\hline 229 & 10.07 & 1198 & 0.57 \\
\hline 267 & 0.62 & 1259 & 4.61 \\
\hline 287 & 0.38 & 1305 & 35.23 \\
\hline 295 & 2.99 & 1308 & 62.15 \\
\hline 364 & 1.12 & 1315 & 999.07 \\
\hline 401 & 0.33 & 1335 & 15.75 \\
\hline 420 & 1.77 & 1350 & 2.48 \\
\hline 434 & 6.33 & 1384 & 0.16 \\
\hline 461 & 9.07 & 1413 & 46.35 \\
\hline 480 & 9.32 & 1445 & 6.22 \\
\hline 497 & 2.19 & 1475 & 13.94 \\
\hline 536 & 15.13 & 1493 & 28.25 \\
\hline 564 & 32.55 & 1510 & 10.77 \\
\hline 569 & 0.72 & 1515 & 4.34 \\
\hline 629 & 4.82 & 1524 & 1.37 \\
\hline 641 & 16.70 & 1527 & 4.70 \\
\hline 701 & 1.42 & 1549 & 1.51 \\
\hline 715 & 2.90 & 1628 & 2.74 \\
\hline 728 & 12.07 & 1637 & 0.26 \\
\hline 750 & 4.39 & 1644 & 0.63 \\
\hline 773 & 74.43 & 1652 & 0.77 \\
\hline 775 & 33.02 & 1692 & 273.54 \\
\hline 778 & 13.17 & 3058 & 27.30 \\
\hline 793 & 3.26 & 3061 & 15.84 \\
\hline 822 & 0.18 & 3098 & 14.52 \\
\hline 835 & 23.26 & 3128 & 27.69 \\
\hline 884 & 0.31 & 3139 & 37.65 \\
\hline 895 & 0.13 & 3191 & 3.48 \\
\hline 910 & 3.88 & 3194 & 0.79 \\
\hline 952 & 0.83 & 3203 & 5.74 \\
\hline 970 & 1.60 & 3206 & 10.31 \\
\hline 991 & 0.06 & 3215 & 19.86 \\
\hline 1006 & 1.02 & 3215 & 20.18 \\
\hline 1018 & 0.05 & 3225 & 11.42 \\
\hline 1025 & 11.60 & 3242 & 7.59 \\
\hline
\end{tabular}


Table S45. B3LYP/6-31G* optimized geometries, energies, and thermal corrections for syn-5-t-butoxycarbonyliminodibenzothiophene 7.

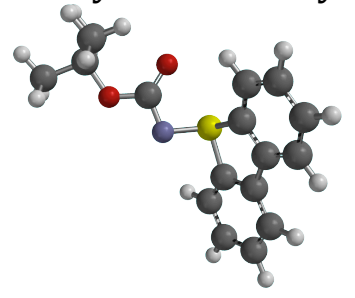

B3LYP/6-31G* energy Zero-point correction Thermal correction to energy
-1182.776940 Hartrees

0.248537685 Hartrees

0.263284060 Hartrees

\begin{tabular}{|c|c|c|c|c|}
\hline \multirow[t]{2}{*}{ Atom } & \multirow{2}{*}{$\begin{array}{l}\text { Atomic } \\
\text { Number }\end{array}$} & \multicolumn{3}{|c|}{ Cartesian Coordinates (Angstroms) } \\
\hline & & $\mathbf{X}$ & $\mathbf{Y}$ & $\mathbf{Z}$ \\
\hline $\mathrm{H}$ & 1 & 0.3674791 & -2.5810075 & -0.7669593 \\
\hline $\mathrm{C}$ & 6 & -0.6513278 & -2.4713672 & -0.4119692 \\
\hline $\mathrm{C}$ & 6 & -3.2975116 & -2.1061672 & 0.5176281 \\
\hline $\mathrm{C}$ & 6 & -1.2272896 & -1.2103149 & -0.3303495 \\
\hline $\mathrm{C}$ & 6 & -1.4206305 & -3.5646438 & -0.0018345 \\
\hline $\mathrm{C}$ & 6 & -2.7309968 & -3.3805929 & 0.4530352 \\
\hline $\mathrm{C}$ & 6 & -2.5379356 & -0.9986345 & 0.1299217 \\
\hline $\mathrm{H}$ & 1 & -0.9958755 & -4.5636000 & -0.0396646 \\
\hline $\mathrm{H}$ & 1 & -3.3183076 & -4.2409720 & 0.7615302 \\
\hline $\mathrm{H}$ & 1 & -4.3176906 & -1.9798094 & 0.8697259 \\
\hline $\mathrm{C}$ & 6 & -2.9102145 & 0.4223580 & 0.1352472 \\
\hline $\mathrm{C}$ & 6 & -3.1816682 & 3.2110979 & 0.0691315 \\
\hline $\mathrm{C}$ & 6 & -1.8730117 & 1.2534521 & -0.3181732 \\
\hline $\mathrm{C}$ & 6 & -4.1091129 & 1.0139644 & 0.5447033 \\
\hline C & 6 & -4.2362122 & 2.4030710 & 0.5082916 \\
\hline C & 6 & -1.9814313 & 2.6361159 & -0.3568753 \\
\hline $\mathrm{H}$ & 1 & -4.9321640 & 0.3997848 & 0.8989228 \\
\hline $\mathrm{H}$ & 1 & -5.1656997 & 2.8627089 & 0.8321959 \\
\hline $\mathrm{H}$ & 1 & -1.1493752 & 3.2479853 & -0.6896967 \\
\hline $\mathrm{H}$ & 1 & -3.2932521 & 4.2912181 & 0.0598805 \\
\hline S & 16 & -0.4475838 & 0.3287219 & -0.8863514 \\
\hline $\mathrm{N}$ & 7 & 0.7399711 & 0.8944939 & 0.1286155 \\
\hline $\mathrm{C}$ & 6 & 1.9014410 & 0.2585879 & -0.2187511 \\
\hline 0 & 8 & 2.0035974 & -0.7177516 & -0.9807072 \\
\hline 0 & 8 & 2.9418760 & 0.8442881 & 0.4154573 \\
\hline $\mathrm{C}$ & 6 & 4.2930924 & 0.2820122 & 0.3413747 \\
\hline $\mathrm{C}$ & 6 & 5.0916881 & 1.2237134 & 1.2480915 \\
\hline $\mathrm{H}$ & 1 & 4.6858752 & 1.2147146 & 2.2648214 \\
\hline $\mathrm{H}$ & 1 & 5.0464011 & 2.2502590 & 0.8702271 \\
\hline $\mathrm{H}$ & 1 & 6.1413011 & 0.9125541 & 1.2874455 \\
\hline $\mathrm{C}$ & 6 & 4.8168175 & 0.3463792 & -1.0979730 \\
\hline $\mathrm{H}$ & 1 & 5.8664211 & 0.0303079 & -1.1265143 \\
\hline $\mathrm{H}$ & 1 & 4.7603960 & 1.3740957 & -1.4738141 \\
\hline $\mathrm{H}$ & 1 & 4.2324756 & -0.3016398 & -1.7527135 \\
\hline $\mathrm{C}$ & 6 & 4.3091906 & -1.1454899 & 0.9006117 \\
\hline $\mathrm{H}$ & 1 & 5.3415380 & -1.5094132 & 0.9596904 \\
\hline $\mathrm{H}$ & 1 & 3.7338930 & -1.8200137 & 0.2647073 \\
\hline $\mathrm{H}$ & 1 & 3.8850193 & -1.1617229 & 1.9108648 \\
\hline
\end{tabular}


Table S46. B3LYP/6-31G* calculated IR frequencies $\left(\mathrm{cm}^{-1}\right.$, unscaled) and intensities for syn-5-t-butoxycarbonyliminodibenzothiophene $\mathbf{7}$.

\begin{tabular}{lrlrlr}
\hline Frequency & Intensity & Frequency & Intensity & Frequency & Intensity \\
\hline 35 & 0.60 & 761 & 3.57 & 1384 & 2.96 \\
50 & 0.21 & 772 & 75.04 & 1418 & 15.37 \\
56 & 0.43 & 777 & 11.06 & 1423 & 40.71 \\
79 & 2.65 & 785 & 33.26 & 1448 & 30.72 \\
98 & 1.24 & 792 & 4.24 & 1475 & 14.47 \\
119 & 1.74 & 818 & 35.69 & 1493 & 27.39 \\
136 & 1.25 & 881 & 0.17 & 1501 & 0.50 \\
150 & 1.43 & 894 & 0.04 & 1511 & 11.43 \\
187 & 1.59 & 898 & 15.86 & 1521 & 0.86 \\
190 & 1.23 & 935 & 0.83 & 1523 & 3.03 \\
228 & 0.21 & 938 & 0.02 & 1524 & 1.02 \\
239 & 8.65 & 950 & 0.85 & 1525 & 22.35 \\
258 & 11.40 & 968 & 1.36 & 1536 & 0.76 \\
267 & 3.19 & 979 & 0.26 & 1559 & 14.52 \\
290 & 3.82 & 991 & 0.06 & 1629 & 2.59 \\
304 & 0.11 & 1003 & 0.63 & 1637 & 0.61 \\
329 & 14.53 & 1018 & 0.02 & 1645 & 1.66 \\
348 & 2.70 & 1050 & 1.38 & 1652 & 2.36 \\
360 & 1.12 & 1057 & 2.41 & 1657 & 166.53 \\
400 & 0.69 & 1064 & 0.29 & 3054 & 12.68 \\
419 & 1.68 & 1067 & 0.30 & 3055 & 22.41 \\
426 & 4.56 & 1073 & 3.61 & 3063 & 37.42 \\
437 & 8.22 & 1093 & 7.83 & 3116 & 11.02 \\
451 & 11.49 & 1099 & 13.07 & 3118 & 22.87 \\
461 & 2.16 & 1152 & 0.97 & 3133 & 53.01 \\
480 & 4.17 & 1160 & 0.89 & 3136 & 30.94 \\
498 & 2.95 & 1196 & 0.61 & 3169 & 5.10 \\
518 & 12.18 & 1198 & 3.61 & 3173 & 12.07 \\
547 & 10.03 & 1221 & 618.44 & 3190 & 3.81 \\
569 & 0.38 & 1259 & 0.98 & 3193 & 0.80 \\
629 & 5.30 & 1282 & 17.19 & 3202 & 5.55 \\
699 & 1.04 & 1290 & 113.58 & 3205 & 12.33 \\
709 & 5.93 & 1308 & 8.20 & 3214 & 22.65 \\
726 & 7.89 & 1334 & 39.13 & 3214 & 19.79 \\
738 & 74.09 & 1349 & 564.01 & 3226 & 8.83 \\
742 & 4.85 & 1351 & 400.28 & 3239 & 6.70 \\
\hline & & & & &
\end{tabular}


Table S47. B3LYP/6-31G* optimized geometries, energies, and thermal corrections for anti-5-t-butoxycarbonyliminodibenzothiophene $\mathbf{7}$.

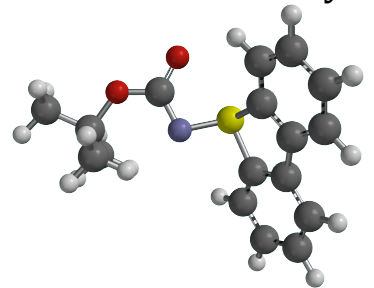

B3LYP/6-31G* energy Zero-point correction Thermal correction to energy
-1261.406954 Hartrees

0.304068068 Hartrees

0.321273556 Hartrees

\begin{tabular}{|c|c|c|c|c|}
\hline \multirow[t]{2}{*}{ Atom } & \multirow{2}{*}{$\begin{array}{c}\text { Atomic } \\
\text { Number }\end{array}$} & \multicolumn{3}{|c|}{ Cartesian Coordinates (Angstroms) } \\
\hline & & $\mathbf{X}$ & $\mathbf{Y}$ & $\mathbf{Z}$ \\
\hline $\mathrm{H}$ & 1 & -0.4047087 & -3.0644583 & -0.7825513 \\
\hline $\mathrm{C}$ & 6 & -1.2854578 & -2.6187402 & -0.3340611 \\
\hline $\mathrm{C}$ & 6 & -3.5451799 & -1.3805848 & 0.8363096 \\
\hline $\mathrm{C}$ & 6 & -1.4370463 & -1.2379560 & -0.3233485 \\
\hline $\mathrm{C}$ & 6 & -2.2876449 & -3.3846115 & 0.2696428 \\
\hline $\mathrm{C}$ & 6 & -3.4061495 & -2.7696924 & 0.8427405 \\
\hline $\mathrm{C}$ & 6 & -2.5456927 & -0.5956142 & 0.2544348 \\
\hline $\mathrm{H}$ & 1 & -2.1949317 & -4.4665355 & 0.2905645 \\
\hline $\mathrm{H}$ & 1 & -4.1789788 & -3.3804436 & 1.3010799 \\
\hline $\mathrm{H}$ & 1 & -4.4202699 & -0.9169207 & 1.2831714 \\
\hline $\mathrm{C}$ & 6 & -2.4682785 & 0.8673847 & 0.1510573 \\
\hline $\mathrm{C}$ & 6 & -1.9109962 & 3.5908501 & -0.2009598 \\
\hline $\mathrm{C}$ & 6 & -1.3017319 & 1.3012237 & -0.4996275 \\
\hline $\mathrm{C}$ & 6 & -3.3700496 & 1.8277882 & 0.6197624 \\
\hline $\mathrm{C}$ & 6 & -3.0850045 & 3.1819846 & 0.4412224 \\
\hline $\mathrm{C}$ & 6 & -1.0052010 & 2.6441701 & -0.6867689 \\
\hline $\mathrm{H}$ & 1 & -4.2816071 & 1.5237996 & 1.1261456 \\
\hline $\mathrm{H}$ & 1 & -3.7834807 & 3.9282265 & 0.8091055 \\
\hline $\mathrm{H}$ & 1 & -0.0891603 & 2.9470524 & -1.1837433 \\
\hline $\mathrm{H}$ & 1 & -1.7004879 & 4.6490180 & -0.3245976 \\
\hline S & 16 & -0.3113583 & -0.0612930 & -1.1156498 \\
\hline $\mathrm{N}$ & 7 & 1.1150792 & 0.1581968 & -0.2749591 \\
\hline $\mathrm{C}$ & 6 & 1.9368053 & -0.8660443 & -0.6674834 \\
\hline 0 & 8 & 1.5904126 & -1.8321678 & -1.3606099 \\
\hline 0 & 8 & 3.2199537 & -0.8327823 & -0.2400378 \\
\hline $\mathrm{C}$ & 6 & 3.8385215 & 0.2297984 & 0.5586220 \\
\hline $\mathrm{C}$ & 6 & 5.2805992 & -0.2729633 & 0.6941721 \\
\hline $\mathrm{H}$ & 1 & 5.7404463 & -0.3855601 & -0.2928264 \\
\hline $\mathrm{H}$ & 1 & 5.2995138 & -1.2457685 & 1.1956777 \\
\hline $\mathrm{H}$ & 1 & 5.8774339 & 0.4344474 & 1.2799819 \\
\hline $\mathrm{C}$ & 6 & 3.1728848 & 0.3257458 & 1.9357531 \\
\hline $\mathrm{H}$ & 1 & 3.1847917 & -0.6528836 & 2.4281072 \\
\hline $\mathrm{H}$ & 1 & 2.1392862 & 0.6648503 & 1.8471585 \\
\hline $\mathrm{H}$ & 1 & 3.7263119 & 1.0316577 & 2.5661547 \\
\hline $\mathrm{C}$ & 6 & 3.8098980 & 1.5638946 & -0.1952539 \\
\hline $\mathrm{H}$ & 1 & 4.2401250 & 1.4426020 & -1.1956300 \\
\hline $\mathrm{H}$ & 1 & 4.4083562 & 2.3060732 & 0.3460077 \\
\hline $\mathrm{H}$ & 1 & 2.7889521 & 1.9379531 & -0.2907987 \\
\hline
\end{tabular}


Table S48. B3LYP/6-31G* calculated IR frequencies $\left(\mathrm{cm}^{-1}\right.$, unscaled) and intensities for anti-5-t-butoxycarbonyliminodibenzothiophene $\mathbf{7}$.

\begin{tabular}{|c|c|c|c|c|c|}
\hline Frequency & Intensity & Frequency & Intensity & Frequency & Intensity \\
\hline 22 & 1.37 & 773 & 69.44 & 1384 & 0.17 \\
\hline 41 & 0.05 & 776 & 31.47 & 1422 & 12.87 \\
\hline 50 & 0.72 & 778 & 15.69 & 1422 & 8.85 \\
\hline 80 & 2.05 & 783 & 1.76 & 1451 & 44.45 \\
\hline 99 & 1.08 & 793 & 3.46 & 1474 & 14.81 \\
\hline 111 & 1.99 & 836 & 30.70 & 1493 & 27.92 \\
\hline 136 & 1.85 & 883 & 1.91 & 1497 & 0.70 \\
\hline 149 & 1.85 & 891 & 12.94 & 1509 & 11.14 \\
\hline 179 & 7.02 & 895 & 0.26 & 1516 & 0.98 \\
\hline 185 & 3.14 & 931 & 0.12 & 1518 & 0.70 \\
\hline 210 & 0.98 & 932 & 1.90 & 1522 & 17.84 \\
\hline 227 & 10.06 & 952 & 1.02 & 1523 & 1.99 \\
\hline 245 & 1.02 & 970 & 1.81 & 1532 & 0.43 \\
\hline 266 & 0.92 & 976 & 0.08 & 1556 & 7.90 \\
\hline 267 & 0.12 & 992 & 0.05 & 1628 & 2.79 \\
\hline 289 & 1.16 & 1006 & 1.41 & 1637 & 0.50 \\
\hline 345 & 2.36 & 1018 & 0.01 & 1644 & 1.86 \\
\hline 351 & 0.69 & 1049 & 2.39 & 1651 & 0.52 \\
\hline 365 & 4.65 & 1057 & 2.02 & 1671 & 271.77 \\
\hline 401 & 0.37 & 1061 & 0.52 & 3053 & 22.30 \\
\hline 405 & 1.41 & 1063 & 6.52 & 3054 & 9.36 \\
\hline 419 & 2.47 & 1072 & 6.07 & 3063 & 38.37 \\
\hline 435 & 9.44 & 1087 & 32.26 & 3116 & 10.03 \\
\hline 461 & 3.09 & 1094 & 1.69 & 3118 & 15.61 \\
\hline 466 & 18.25 & 1151 & 0.94 & 3132 & 45.50 \\
\hline 479 & 7.68 & 1159 & 1.45 & 3135 & 29.27 \\
\hline 497 & 1.44 & 1196 & 1.11 & 3163 & 1.09 \\
\hline 540 & 9.29 & 1197 & 5.07 & 3166 & 26.05 \\
\hline 562 & 20.61 & 1214 & 460.09 & 3192 & 3.22 \\
\hline 569 & 0.72 & 1259 & 0.65 & 3195 & 0.85 \\
\hline 625 & 35.50 & 1277 & 15.03 & 3204 & 6.28 \\
\hline 629 & 5.52 & 1287 & 2.68 & 3206 & 9.24 \\
\hline 700 & 1.32 & 1307 & 10.05 & 3215 & 19.18 \\
\hline 713 & 2.97 & 1331 & 528.45 & 3215 & 22.83 \\
\hline 727 & 12.77 & 1336 & 241.63 & 3224 & 11.54 \\
\hline 749 & 4.95 & 1350 & 3.25 & 3244 & 7.71 \\
\hline
\end{tabular}


Table S49. B3LYP/6-31G* optimized geometries, energies, and thermal corrections for ethoxycarbonylnitrene-acetonitrile biradical.

B3LYP/6-31G* energy

Zero-point correction

Thermal correction to energy
-455.153864 Hartrees

0.130229118 Hartrees

0.140083318 Hartrees

\begin{tabular}{lrrrr}
\hline Atom & $\begin{array}{c}\text { Atomic } \\
\text { Number }\end{array}$ & \multicolumn{2}{c}{ Cartesian Coordinates (Angstroms) } \\
& $\mathbf{X}$ & $\mathbf{Y}$ & \multicolumn{1}{c}{$\mathbf{Z}$} \\
\hline $\mathrm{H}$ & 1 & -3.90233 & 0.486008 & 1.154261 \\
$\mathrm{C}$ & 6 & -3.64928 & 0.869248 & 0.160923 \\
$\mathrm{H}$ & 1 & -4.16547 & 0.258728 & -0.58646 \\
$\mathrm{H}$ & 1 & -4.01385 & 1.899188 & 0.080735 \\
$\mathrm{C}$ & 6 & -2.14849 & 0.83769 & -0.06318 \\
$\mathrm{H}$ & 1 & -1.61923 & 1.436645 & 0.685204 \\
$\mathrm{H}$ & 1 & -1.87742 & 1.215949 & -1.0533 \\
$\mathrm{O}$ & 8 & -1.73932 & -0.54851 & 0.047544 \\
$\mathrm{C}$ & 6 & -0.4465 & -0.89932 & -0.04556 \\
$\mathrm{O}$ & 8 & -0.09621 & -2.05399 & 0.042079 \\
$\mathrm{~N}$ & 7 & 0.416179 & 0.200715 & -0.34856 \\
$\mathrm{~N}$ & 7 & 1.665276 & -0.06481 & 0.019486 \\
$\mathrm{C}$ & 6 & 2.619763 & 0.758182 & -0.19212 \\
$\mathrm{C}$ & 6 & 4.035977 & 0.582958 & 0.206555 \\
$\mathrm{H}$ & 1 & 4.328013 & 1.391223 & 0.889305 \\
$\mathrm{H}$ & 1 & 4.681857 & 0.672874 & -0.67557 \\
$\mathrm{H}$ & 1 & 4.213574 & -0.38442 & 0.69266 \\
\hline
\end{tabular}


Table S50. B3LYP/6-31G* calculated IR frequencies $\left(\mathrm{cm}^{-1}\right.$, unscaled) and intensities for ethoxycarbonylnitrene-acetonitrile biradical.

\begin{tabular}{rrrr}
\hline Frequency & Intensity & Frequency & Intensity \\
\hline 32 & 1.9 & 1178 & 6.67 \\
41 & 0.27 & 1187 & 4.83 \\
68 & 2.22 & 1268 & 717.35 \\
113 & 7.87 & 1314 & 2.46 \\
121 & 0.6 & 1410 & 10.09 \\
165 & 0.81 & 1412 & 3.38 \\
192 & 3.37 & 1446 & 17.02 \\
257 & 0.49 & 1480 & 33.28 \\
287 & 0.28 & 1482 & 41.95 \\
322 & 2.52 & 1513 & 5.31 \\
351 & 1.92 & 1525 & 6.06 \\
470 & 8.46 & 1544 & 8.15 \\
586 & 5.33 & 1578 & 112.45 \\
614 & 38.66 & 1766 & 258.86 \\
720 & 18.84 & 3034 & 4.86 \\
825 & 0.71 & 3063 & 18.01 \\
859 & 9.46 & 3071 & 21.17 \\
939 & 0.93 & 3101 & 3.37 \\
971 & 6.22 & 3113 & 6.52 \\
1011 & 4.1 & 3116 & 7.54 \\
1066 & 42.25 & 3135 & 23.92 \\
1130 & 12.66 & 3146 & 35.98 \\
\hline
\end{tabular}


Table S51. B3LYP/6-31G* optimized geometries, energies, and thermal corrections for acetonitrile.

B3LYP/6-31G* energy

Zero-point correction

Thermal correction to energy
-132.754941 Hartrees

0.045641675 Hartrees

0.050181496 Hartrees

\begin{tabular}{|c|c|c|c|c|}
\hline \multirow[t]{2}{*}{ Atom } & \multirow[t]{2}{*}{ number } & \multicolumn{3}{|c|}{ Cartesian Coordinates (Angstroms) } \\
\hline & & $\mathbf{X}$ & $\mathbf{Y}$ & $\mathbf{Z}$ \\
\hline $\mathrm{H}$ & 1 & 1.0266582 & 0 & 1.5606633 \\
\hline $\mathrm{C}$ & 6 & 0.0000000 & 0 & 1.1809798 \\
\hline $\mathrm{H}$ & 1 & -0.5133291 & -0.889112 & 1.5606633 \\
\hline $\mathrm{H}$ & 1 & -0.5133291 & 0.889112 & 1.5606633 \\
\hline $\mathrm{C}$ & 6 & 0 & 0 & -0.2804904 \\
\hline $\mathrm{N}$ & 7 & 0 & 0 & -1.4407037 \\
\hline
\end{tabular}

Table S52. B3LYP/6-31G* calculated IR frequencies $\left(\mathrm{cm}^{-1}\right.$, unscaled) and intensities for acetonitrile.

\begin{tabular}{rrrr}
\hline Frequency & Intensity & Frequency & Intensity \\
\hline 387 & 0.56 & 1502 & 10.11 \\
387 & 0.56 & 1502 & 10.1 \\
95 & 1.88 & 2382 & 7.36 \\
1076 & 3.31 & 3067 & 3.21 \\
1076 & 3.3 & 3141 & 1.97 \\
1439 & 2.35 & 3141 & 1.97 \\
\hline
\end{tabular}


Figure S1. Energy profile for the rotation of C-O bond of singlet ethoxycarbonylnitrene $\mathbf{2 s}$.

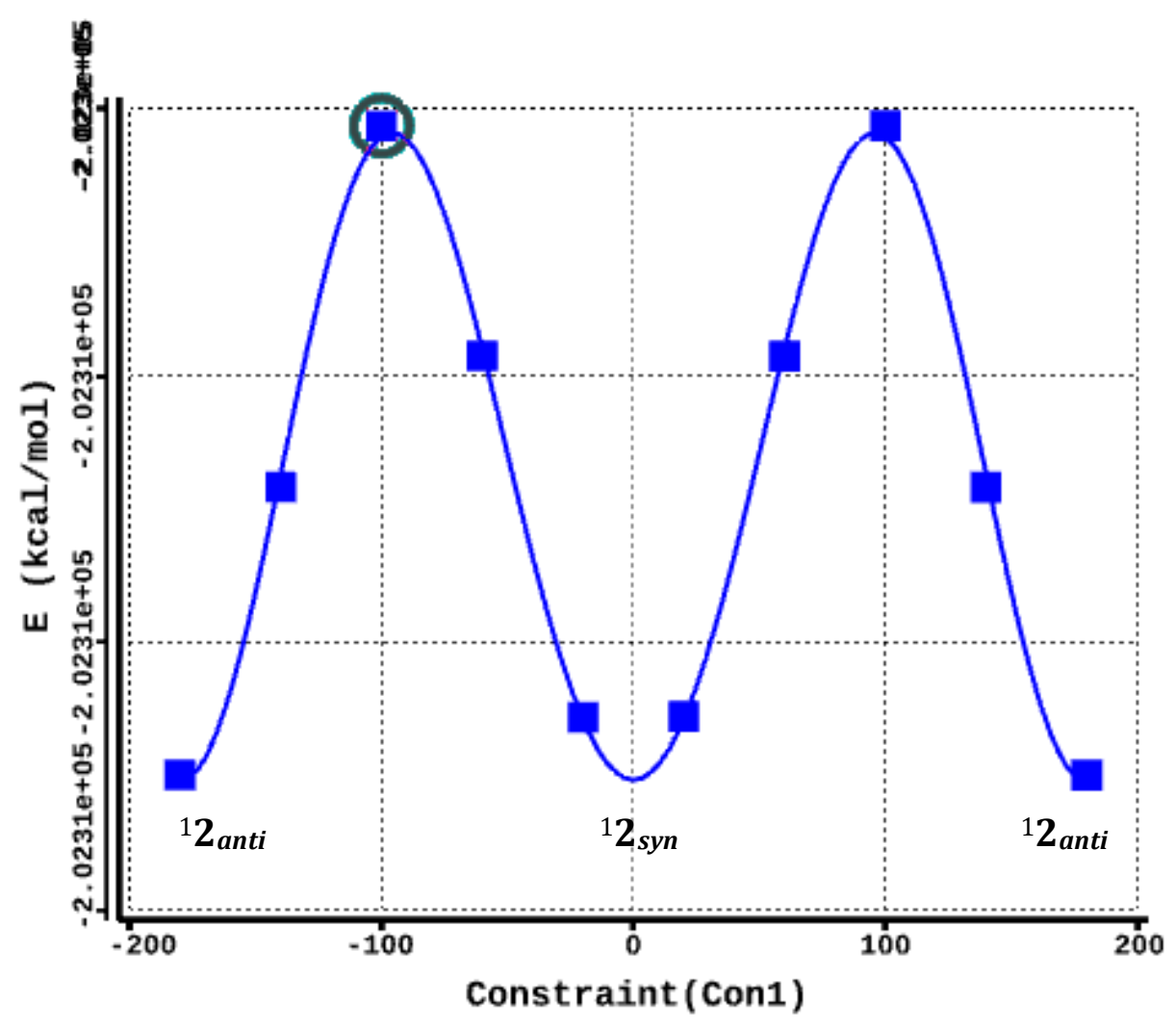


Figure S2. Energy profile for the rotation of C-O bond of triplet ethoxycarbonylnitrene $\mathbf{2 t}$.

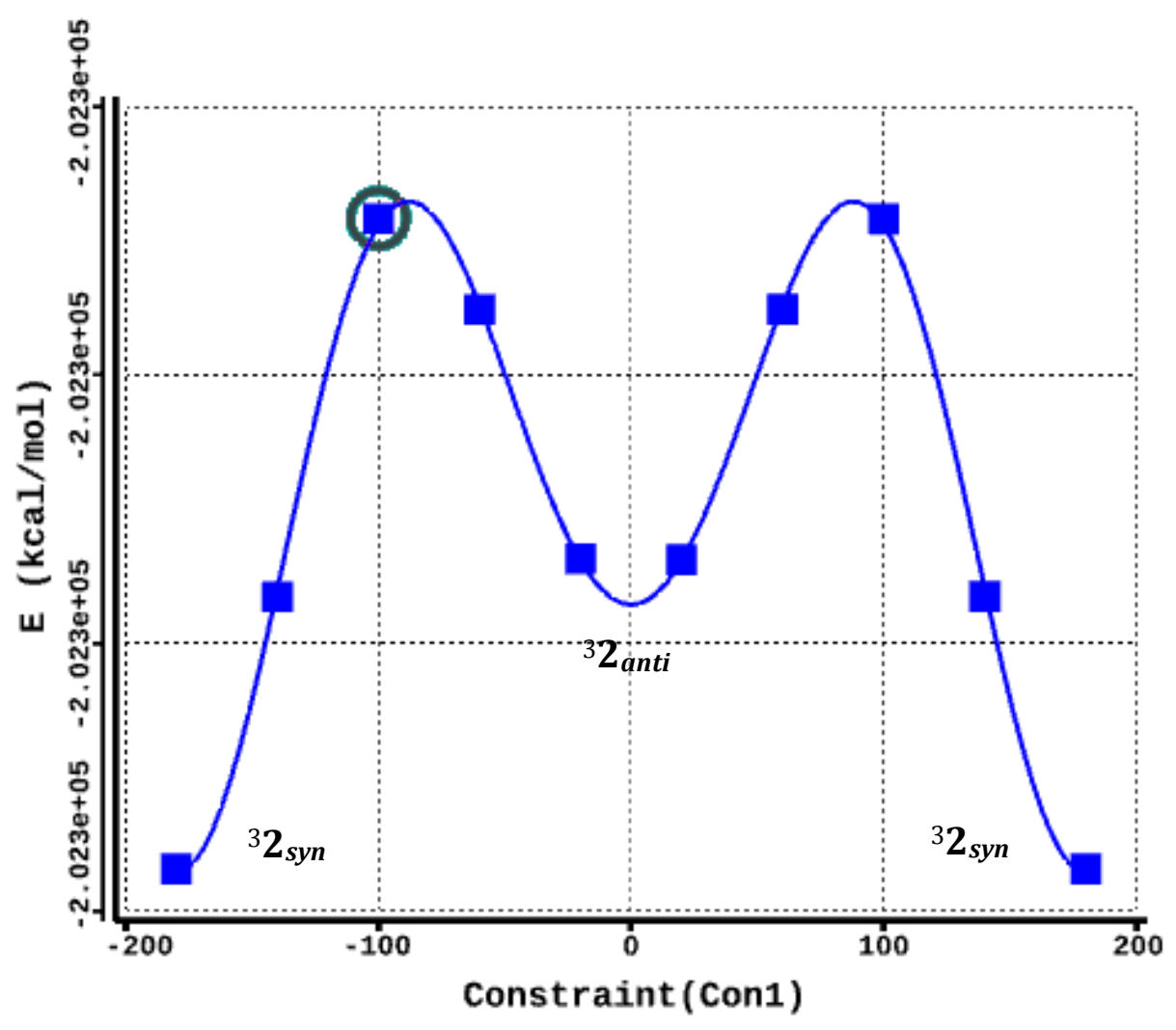


Figure S3. Energy profile for the rotation of C-O bond of singlet $t$-butoxycarbonylnitrene $\mathbf{4 s .}$

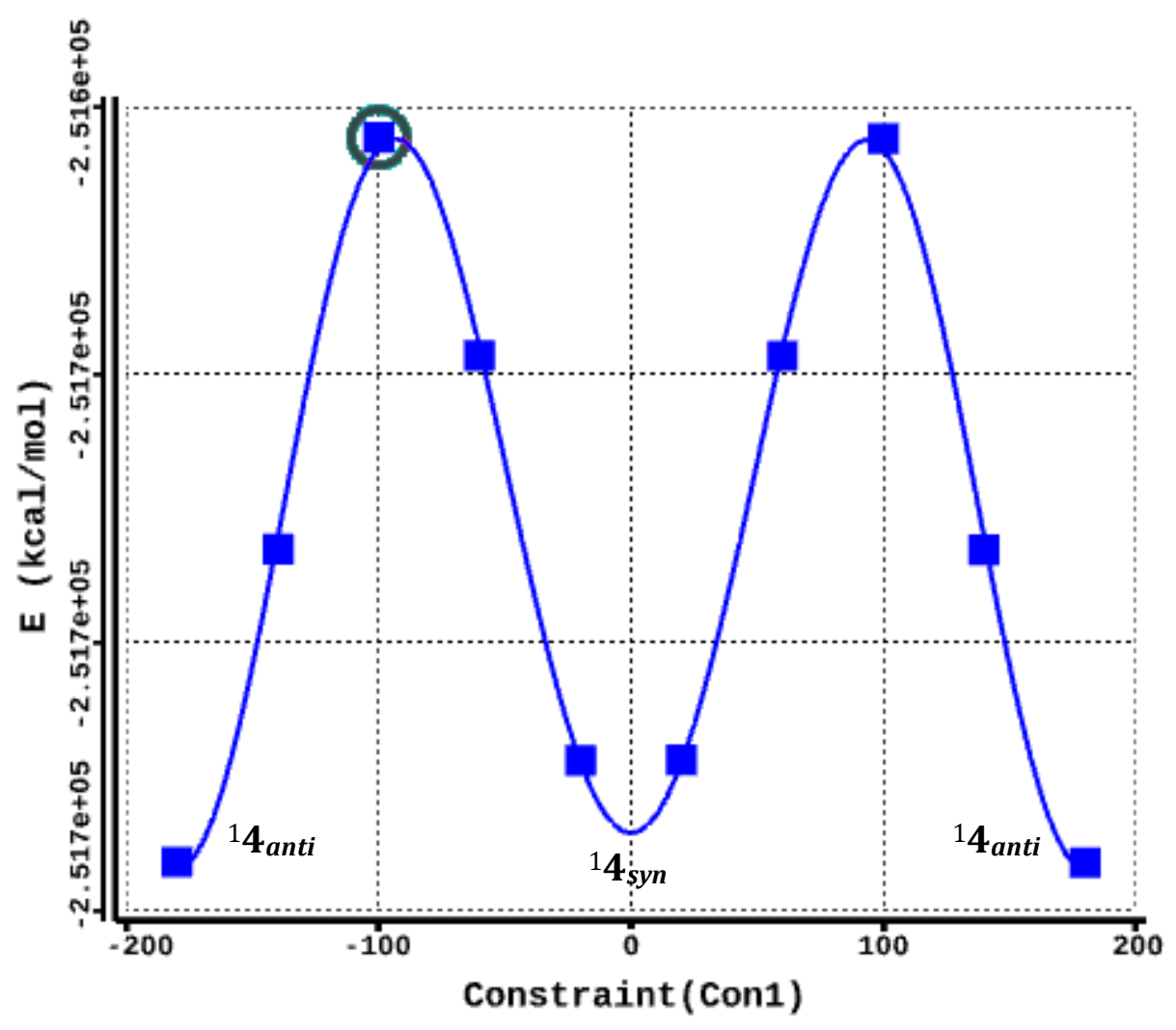


Figure S4. Energy profile for the rotation of C-O bond of triplet t-butoxycarbonylnitrene $\mathbf{4 t}$.
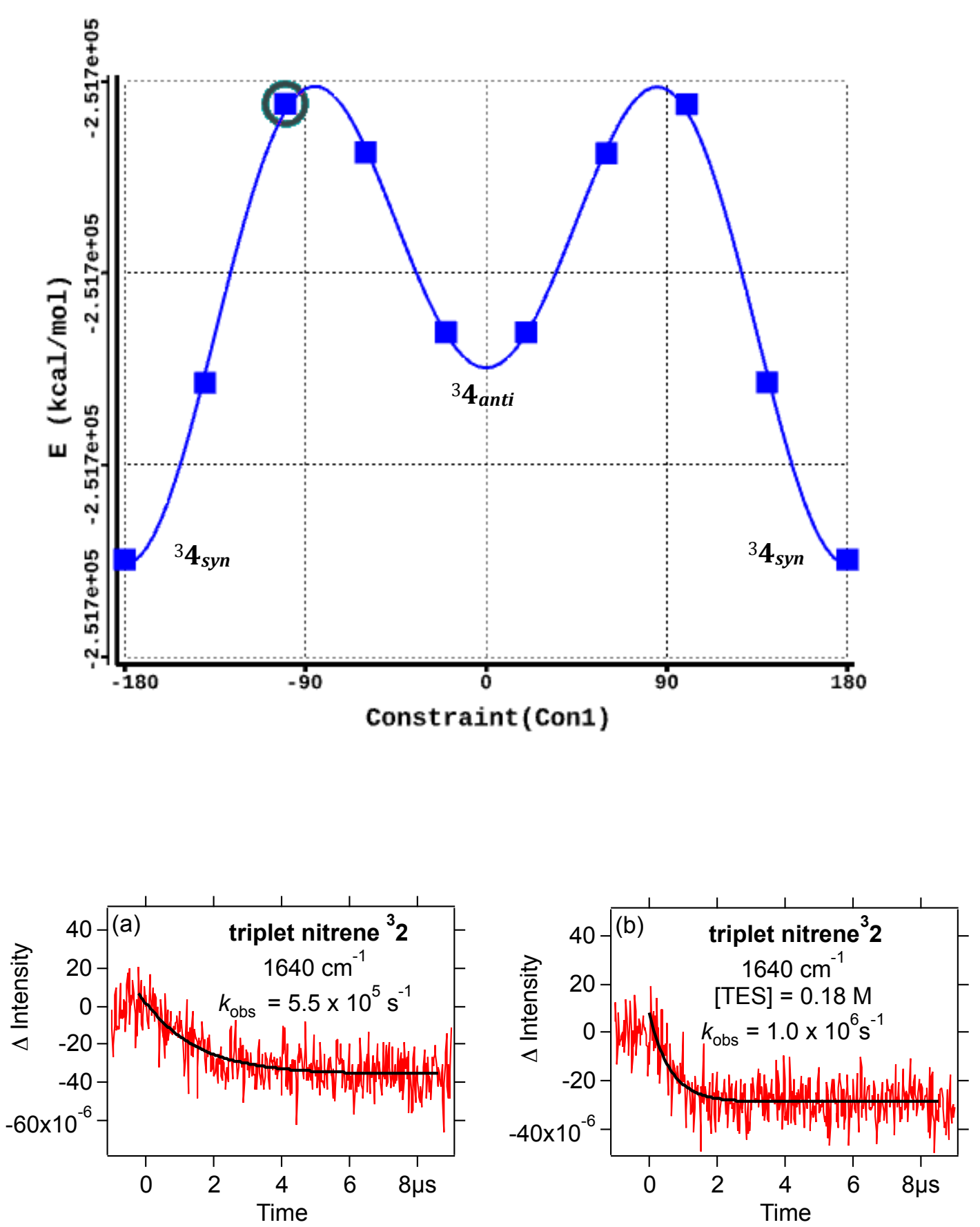

Figure S5. Kinetic traces observed at $1640 \mathrm{~cm}^{-1}$ following $266 \mathrm{~nm}$ laser photolysis of of $6(3 \mathrm{mM})$ in argon-saturated acetonitrile (a) without or (b) with triethylsilane (TES) in presence. The dotted curves are experimental data; the solid curves are best fits to a single-exponential function. 
Compound characterization: ${ }^{1} \mathrm{H}$ and ${ }^{13} \mathrm{C}$ NMR spectra
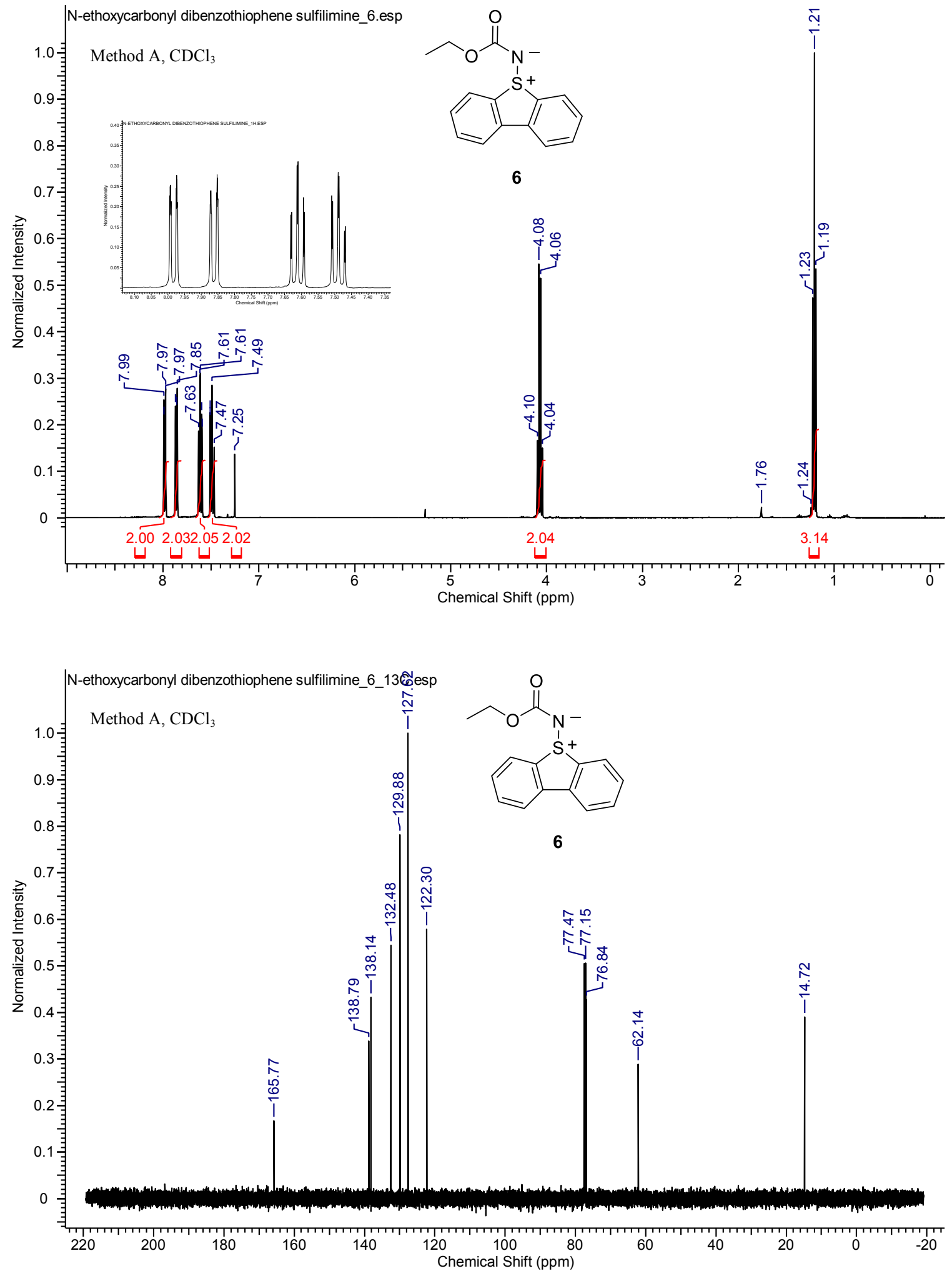

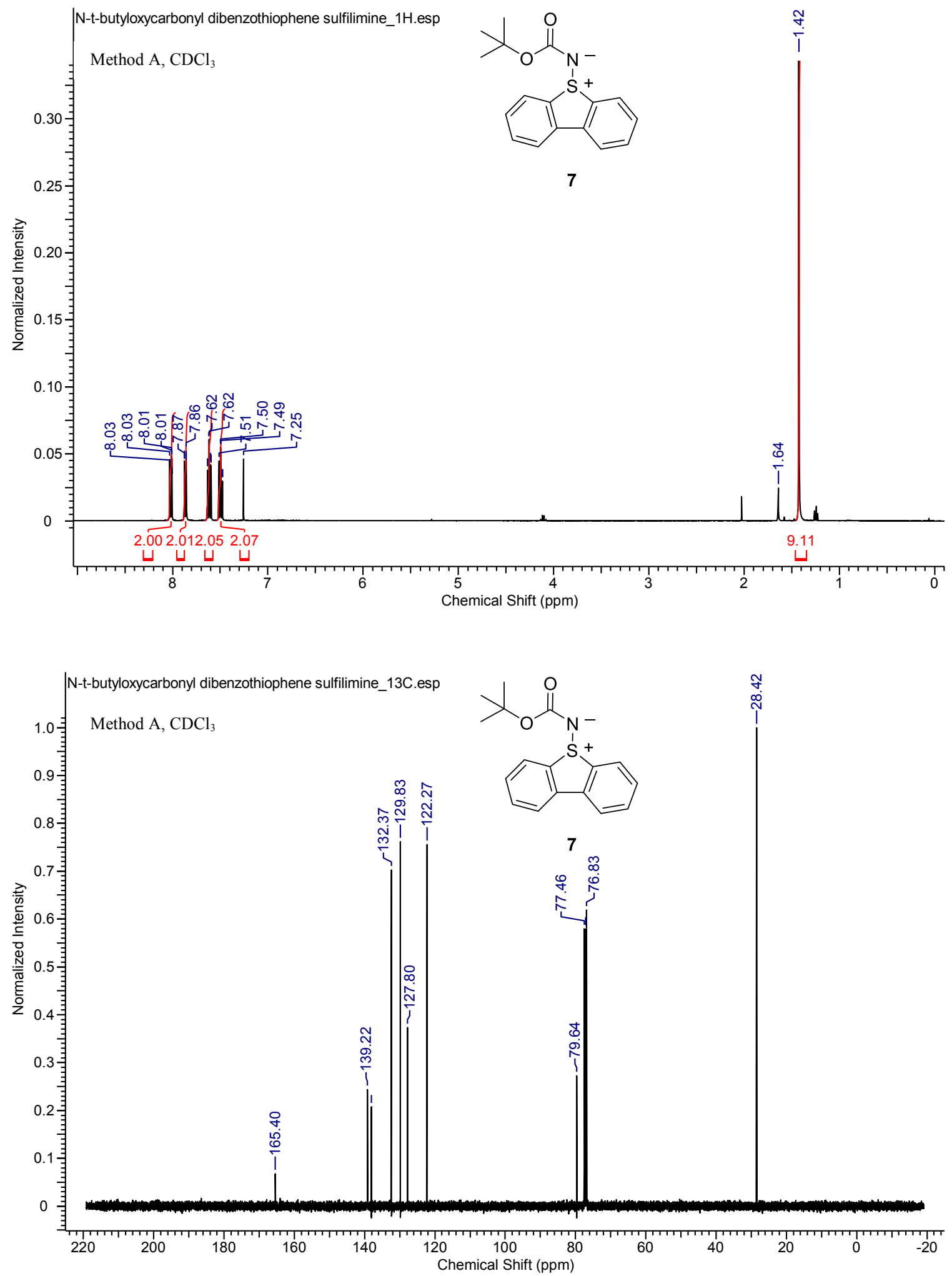
Photolysis product studies: ${ }^{1} \mathrm{H}$ NMR spectra of the photolysis of $\mathbf{6}$ and $\mathbf{7}$

\section{Initial, $\mathrm{CD}_{3} \mathrm{CN}$}

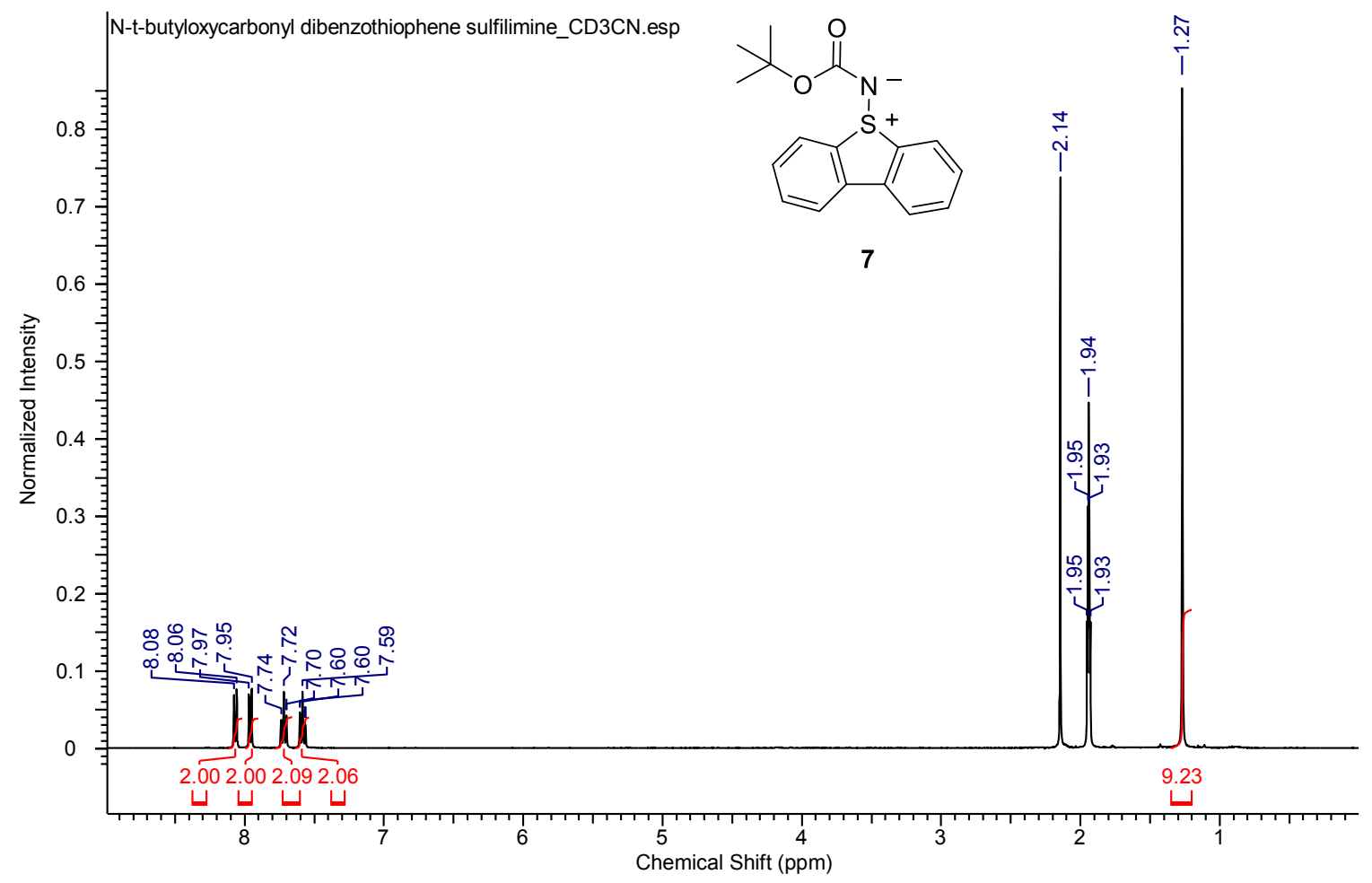

7 Initial, $\mathrm{CD}_{2} \mathrm{Cl}_{2}$

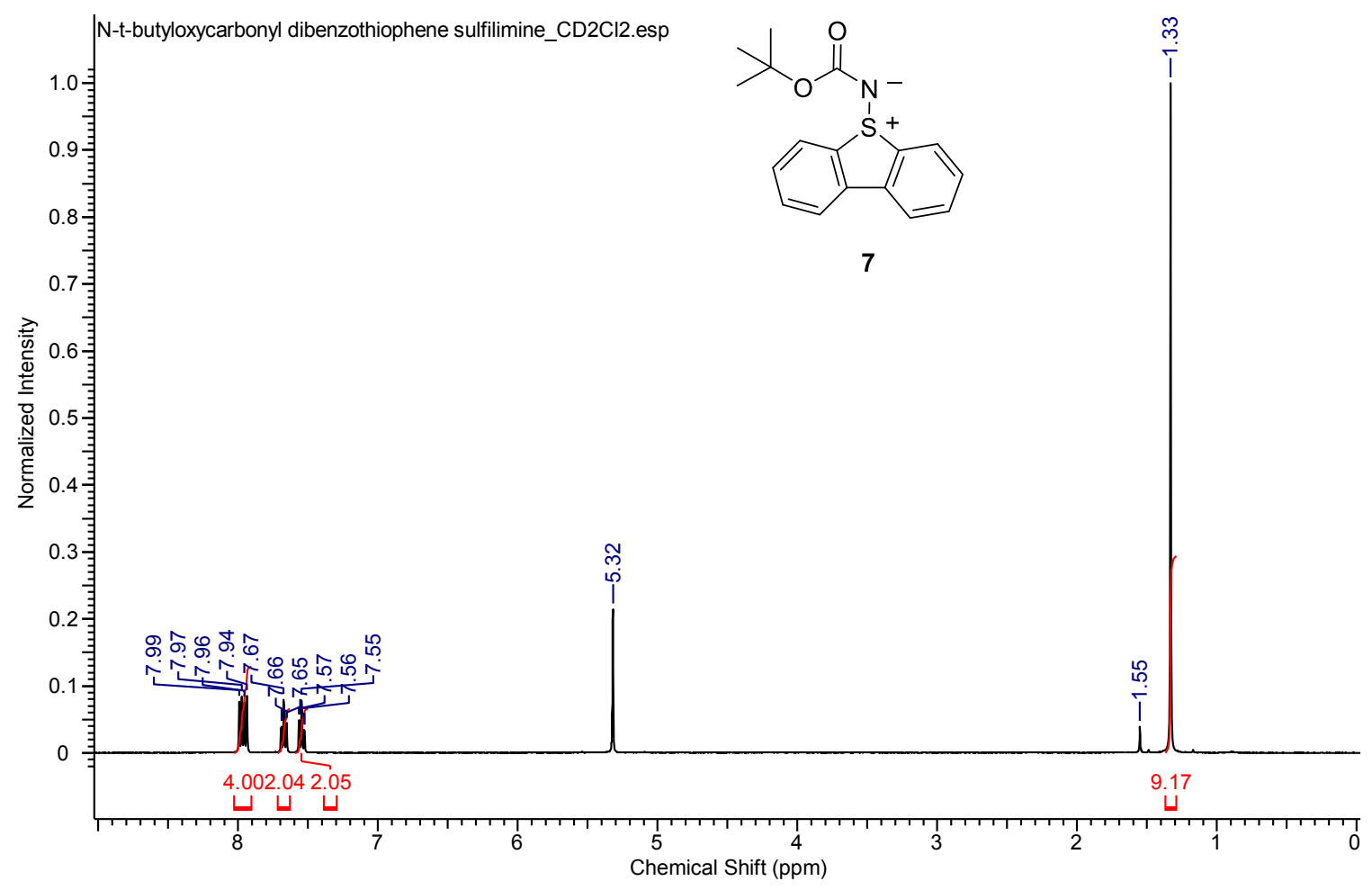


7 After 4 hour photolysis at $254 \mathrm{~nm}, \mathrm{CD}_{3} \mathrm{CN}$

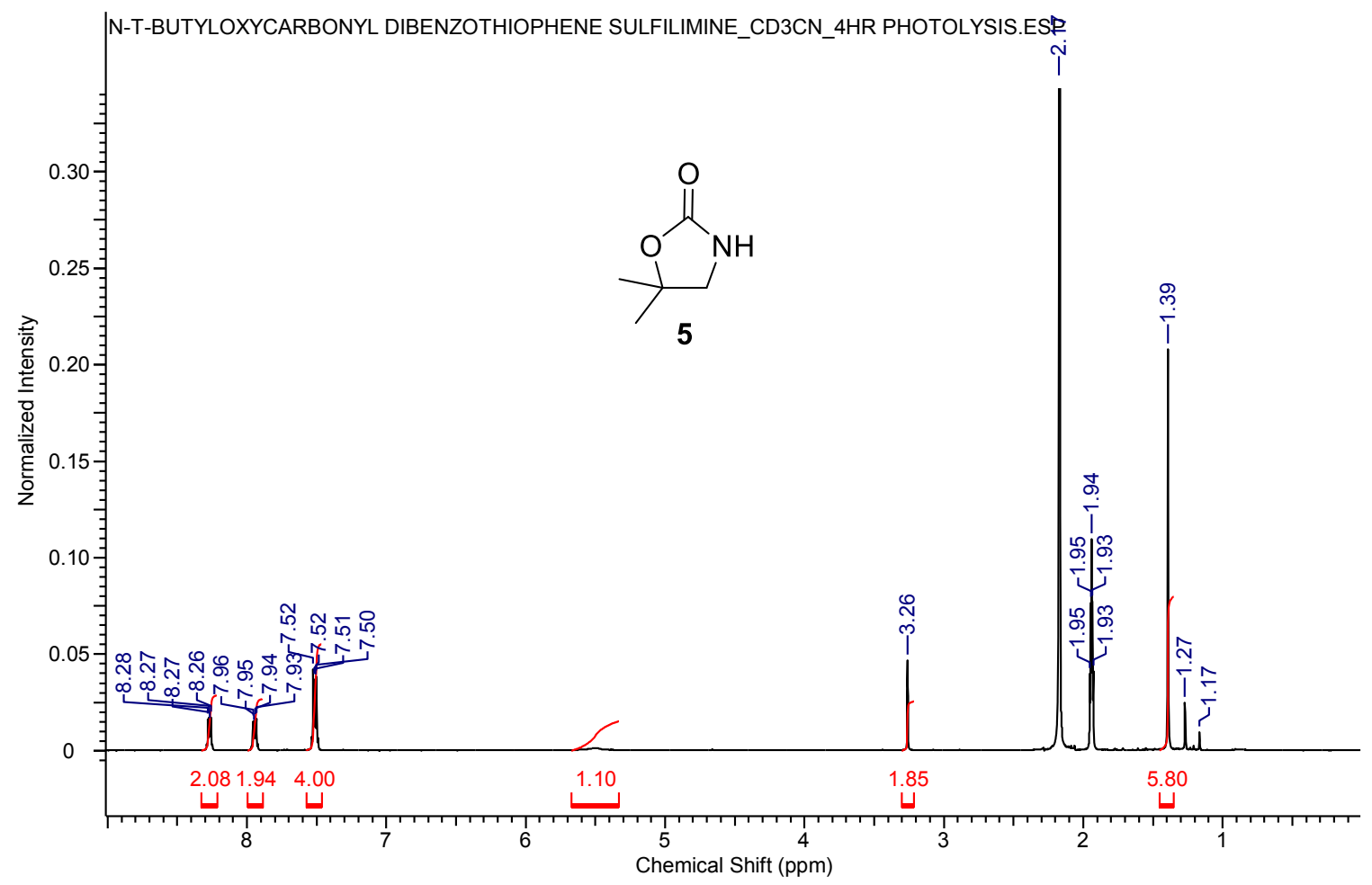

\section{After 4 hour photolysis at $254 \mathrm{~nm}, \mathrm{CD}_{2} \mathrm{Cl}_{2}$}

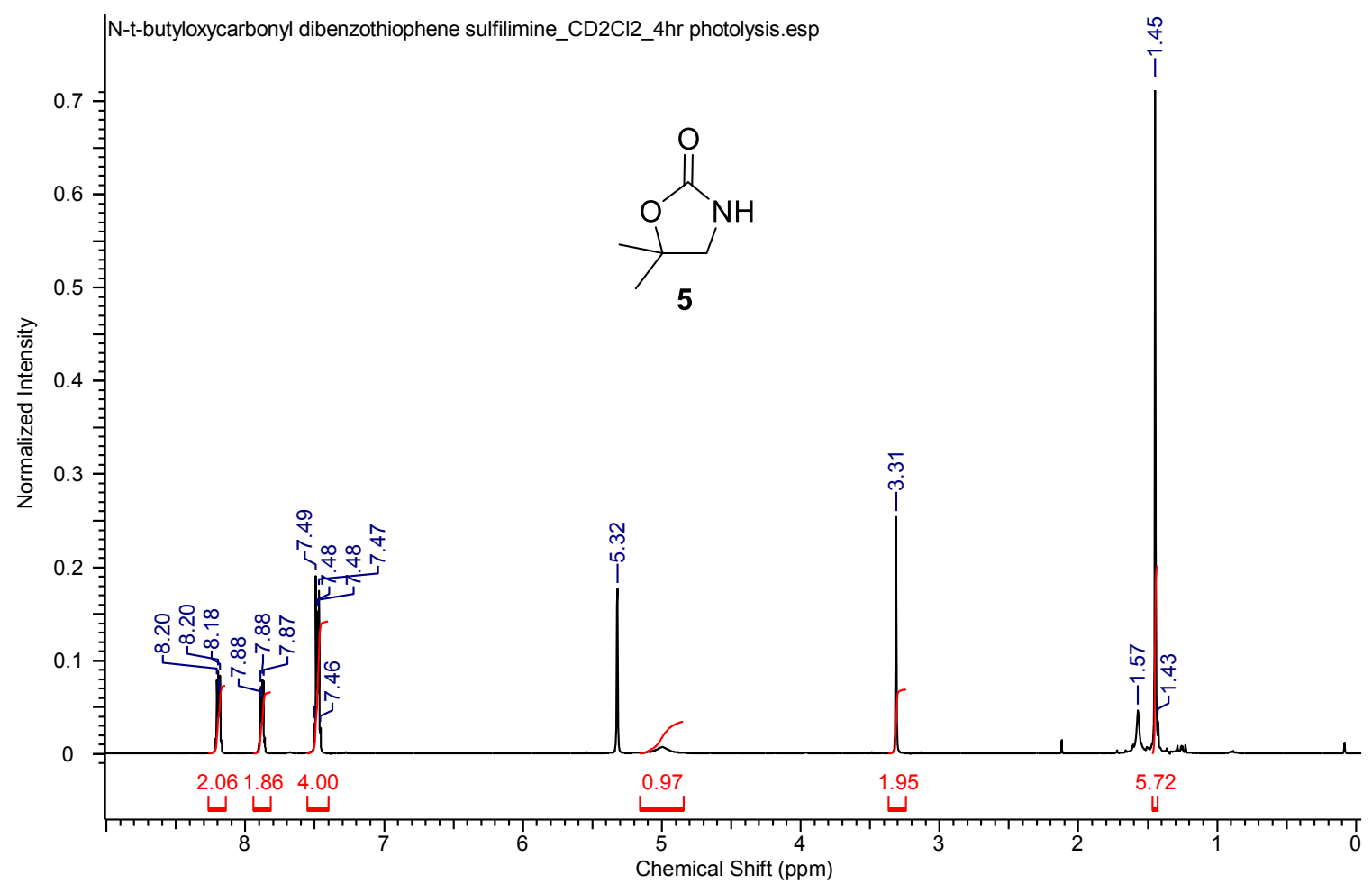


6 After 4 hour photolysis at $254 \mathrm{~nm}, \mathrm{CD}_{3} \mathrm{CN}$

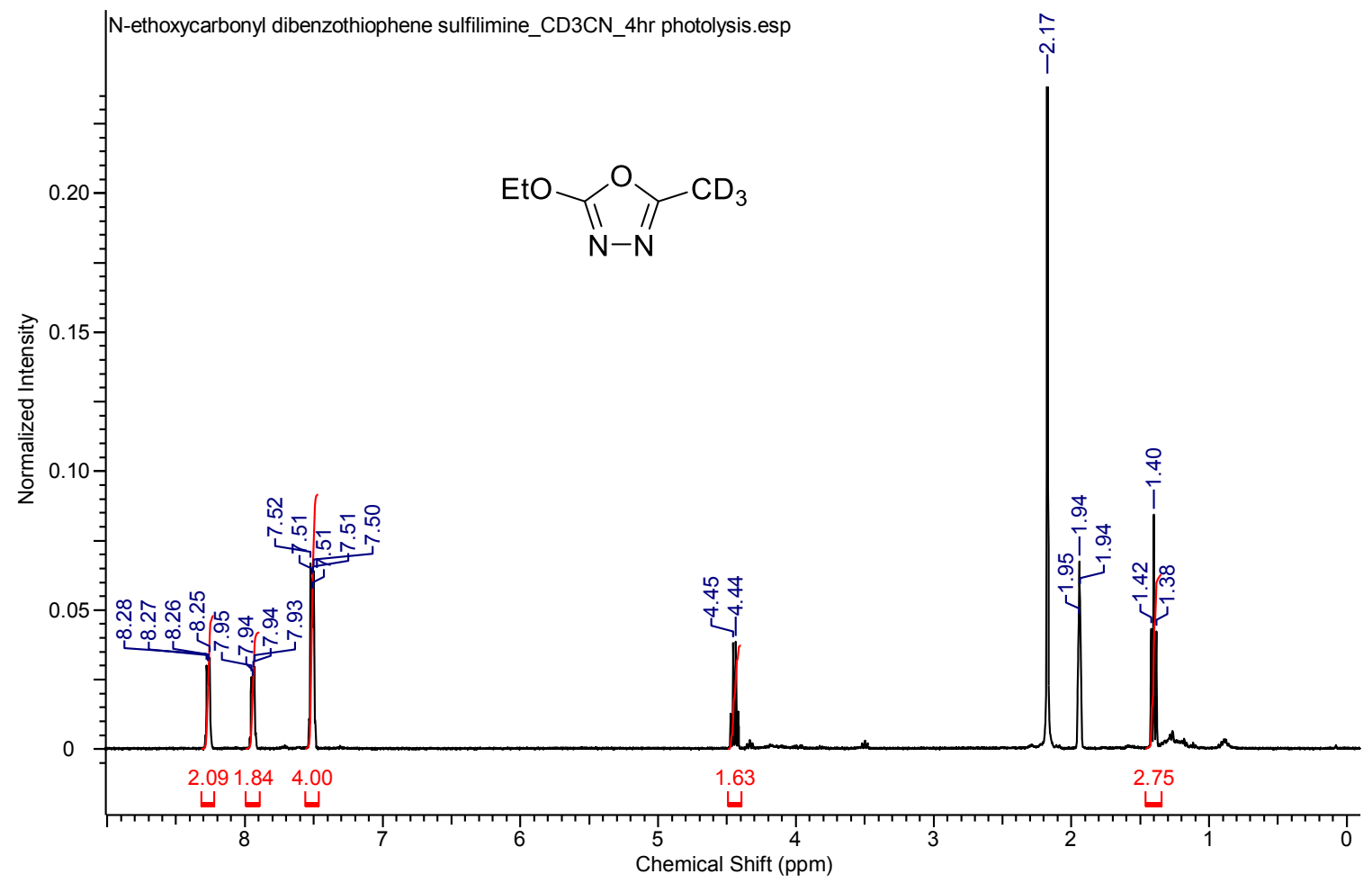

6 After 4 hour photolysis at $254 \mathrm{~nm}$ in $\mathrm{CH}_{3} \mathrm{CN}$, NMR in $\mathrm{CD}_{3} \mathrm{CN}$

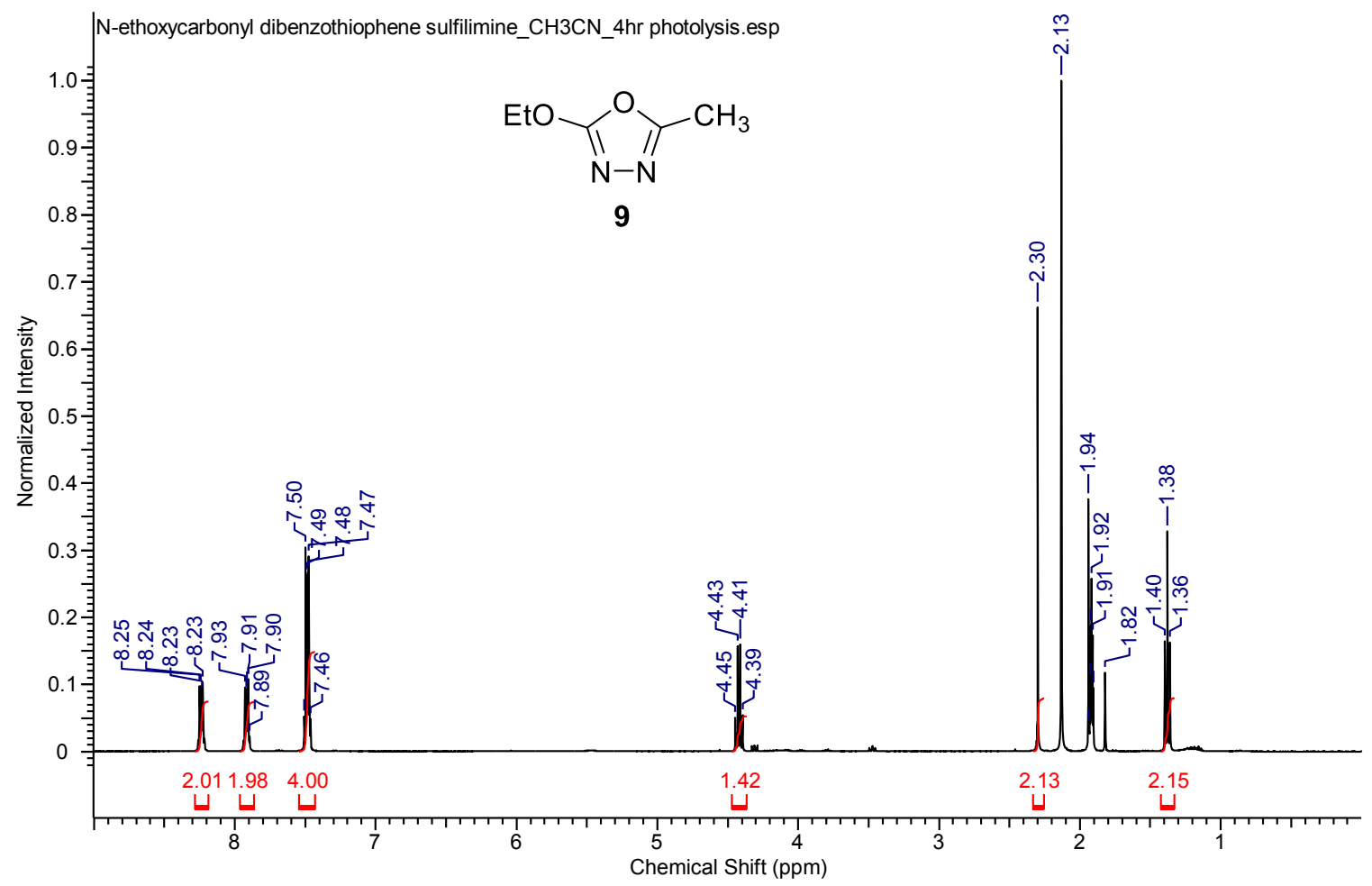

